\title{
Anti-PD-1/PD-L1 Therapy for Non-Small-Cell Lung Cancer: Toward Personalized Medicine and Combination Strategies
}

\author{
Hongshu Sui, ${ }^{1}$ Ningxia Ma $\mathbb{D}^{2},{ }^{2}$ Ying Wang, ${ }^{2}$ Hui Li, ${ }^{2}$ Xiaoming Liu $\mathbb{D}^{2},{ }^{2}$ Yanping Su $\mathbb{D},{ }^{1}$ \\ and Jiali Yang $\mathbb{1}^{2}$ \\ ${ }^{1}$ Department of Histology and Embryology, Taishan Medical University, Taian, Shandong 271000, China \\ ${ }^{2}$ Ningxia Human Stem Cell Institute, General Hospital of Ningxia Medical University, Yinchuan, Ningxia 750004, China \\ Correspondence should be addressed to Yanping Su; ypsu@tsmc.edu.cn and Jiali Yang; yangjiali_123@163.com
}

Received 30 November 2017; Revised 24 May 2018; Accepted 26 June 2018; Published 8 August 2018

Academic Editor: Bogdan Kolarz

Copyright (C) 2018 Hongshu Sui et al. This is an open access article distributed under the Creative Commons Attribution License, which permits unrestricted use, distribution, and reproduction in any medium, provided the original work is properly cited.

\begin{abstract}
Lung cancer remains a leading cause of cancer-related mortality worldwide with the poor prognosis. Encouragingly, immune checkpoint blockade targeting programmed death-1 (PD-1) and programmed death-ligand 1 (PD-L1) has dramatically changed the landscape for treatments in patients with non-small-cell lung cancer (NSCLC). However, only a small proportion of NSCLC patients responded to monotherapy of anti-PD-1/PDL1 agents; together, the development of resistance to anti-PD-1/PD-L1 therapy that leads to failure of anti-PD-1/PD-L1 therapy has significantly limited a broad applicability of the findings in clinical practices. Nowadays, several companion diagnostic assays for PDL1 expression have been introduced for identifying patients who may benefit the immunotherapy. In addition, results from clinical trials explored combinatory therapeutic strategies with conventional and/or targeted therapy reported a higher efficacy with an acceptable safety profile in NSCLC treatments, as compared to the monotherapy of these agents alone. In this review article, we summarized several anti-PD-1/PD-L1 agents licensed for NSCLC treatment, with a focus on predictive biomarkers and companion diagnostic assays for identification of NSCLC patients for immunotherapy anti-PD-1/PDL1 antibodies. Of a great interest, potentials of the combinatory therapy of anti-PD-1/PDL1 therapy with a conventional or targeted therapy, or other immunotherapy such as CAR-T cell therapy were emphasized in the article.
\end{abstract}

\section{Introduction}

Lung cancer is still main leading cause of cancer-related mortality worldwide with the poor prognosis. The nonsmall-cell lung (NSCLC) accounts for $\sim 85 \%$ of all patients with lung cancer, and 15\%-30\% of NSCLC are lung squamous cell carcinoma (SQC) [1]. Over the past few decades, the conventional therapeutics (such as surgical resection, chemotherapy, and/or radiotherapy) has been used for treating advanced NSCLC patient. To date, the platinum-based chemotherapy still serves as the first-line therapeutic agent for lung cancer, with a median survival rate of approximately 9-12 months [2]. The therapeutic efficacy has been significantly improved with the introduction of targeted therapies, such as epidermal growth factor receptor tyrosine kinase inhibitors (TKIs) (gefitinib, erlotinib, afatinib, and osimertinib) and anaplastic lymphoma kinase (ALK) inhibitors alectinib, crizotinib, and ceritinib, as evaluated by studies including ALEX study, J-ALEX study [3, 4], ASCEND study [5, 6] PROFILE study [7], and ALUR study [8]. However, these targeted therapies only show excellent initial clinical responses to advance the lifetime of NSCLC patients; the development of resistance limits the therapeutic efficacy of these agents $[9,10]$. Therefore, novel treatment strategies or agents are unmet need to improve the survival rate in NSCLC patients. Encouragingly, immune checkpoint blockade therapy is one of the most successful and exciting clinical benefits in advanced NSCLC [11].

Immune checkpoint inhibitor (ICI) is designed to target an inhibitory immune checkpoint molecule, such as programmed death-ligand 1 (PD-L1) and its receptor, programmed death-1 
TABLE 1: The PD-1/PD-L1 inhibitors licensed for clinical use or under clinical trials for 1062 NSCLC treatment.

\begin{tabular}{|c|c|c|c|c|}
\hline Checkpoint & Blocking agent & $\begin{array}{l}\text { IgG isotype and } \\
\text { characteristics }\end{array}$ & Clinical stage & Manufacturer \\
\hline \multirow{5}{*}{ PD-1 } & $\begin{array}{l}\text { Pembrolizumab (MK3475, } \\
\text { Keytruda, lambrolizumab) }\end{array}$ & Humanized IgG4 mAb & $\begin{array}{c}\text { EMA, FDA approved for } \\
\text { second-line NSCLC } \\
\text { treatment }\end{array}$ & Merck \\
\hline & $\begin{array}{c}\text { Nivolumab (BMS936558, } \\
\text { Opdivo, MDX-1106, ONO-4538) }\end{array}$ & Fully human IgG4 mAb & $\begin{array}{l}\text { FDA approved for first-line } \\
\text { and second-line NSCLC }\end{array}$ & Bristol-Myers Squibb \\
\hline & MEDI0680 (AMP-514) & Humanized IgG4 mAb & Phase I & Medimmune \\
\hline & PDR001 & Humanized IgG4 mAb & Phase I & Novartis \\
\hline & REGN2810 & Humanized IgG4 mAb & Phase I & Regeneron-Sanofi \\
\hline \multirow{4}{*}{ PD-L1 } & $\begin{array}{l}\text { Atezolizumab (Tecentriq, } \\
\text { MPDL3280A, RG7446) }\end{array}$ & High-affinity human IgG1 & $\begin{array}{l}\text { FDA approved for second- } \\
\text { line NSCLC }\end{array}$ & Genentech/Roche \\
\hline & $\begin{array}{l}\text { Durvalumab (MEDI4736, } \\
\text { Infinzi) }\end{array}$ & Human IgG1- $\kappa \mathrm{mAb}$ & $\begin{array}{l}\text { FDA approved for } \\
\text { treatment of unresectable } \\
\text { stage III NSCLC without } \\
\text { relapse after platinum- } \\
\text { based chemoradiation }\end{array}$ & MedImmune/AstraZeneca \\
\hline & BMS-936559 (MDX1105) & $\begin{array}{l}\text { Fully high-affinity human } \\
\text { IgG4 }\end{array}$ & Phase I & Bristol-Myers Squibb \\
\hline & $\begin{array}{l}\text { Avelumab (Bavencio, } \\
\text { MSB0010718C) }\end{array}$ & Fully human IgG1 mAb & $\begin{array}{l}\text { FDA-approved treatment } \\
\text { for metastatic MCC }\end{array}$ & Merck Serono \\
\hline
\end{tabular}

FDA: Food and Drug Administration; Ig: immunoglobulin; mAb: monoclonal antibody; NSCLC: non-small-cell lung cancer; PD-1: programmed death-1; PD-L1: programmed death-ligand 1; PD-L2: programmed death-ligand 2.

(PD-1), or cytotoxic T-lymphocyte-associated antigen 4 (CTLA-4) (Table 1) [12]. Agents targeting the PD-1/PD-L1 signaling have shown promising response in NSCLC treatment. Two antibodies (nivolumab and pembrolizumab) to PD-1 and two antibodies (atezolizumab and durvalumab) to PD-L1 have been approved by the US Food and Drug Administration (FDA) and/or European Medicines Agency (EMA) for treatment of NSCLC (Table 1) [13-16]. Unfortunately, only approximately $20 \%$ patients have positively response to ICIs as monotherapy for NSCLC. Therefore, it is of importance to identify patients who may benefit for immune checkpoint blockade therapy. Currently, four immunohistochemistry (IHC) assays (22C3, 28-8, E1L3N, and SP124) have been registered by FDA as companion and complementary diagnostic assays for detecting the expression of PD-L1 in practice (Table 2). The introduction of these assays has significantly increased the benefit of anti-PD-1/PD-L1 treatments [17], albeit many challenges in anti-PD-1/PD-L1 therapy remain to be overcome. In present review article, the characteristics of anti-PD-1/PD-L1 antibodies, biomarkers, and companion diagnostic assays for patient identification and the significance of the correlation between PD-1/PD-L1 signaling and other driver oncogenes (EGFR, ALK, KRAS, MET, $R O S 1)$ in a combinatory therapy including immune checkpoint blockades and targeted agents were also highlighted.

\section{Companion and Complementary Diagnostic Assays for Precision Medicine of PD-1/PD-L1 Inhibitors}

In the tumor environment, a binding of PD-1 and PD-L1 suppresses the activated $\mathrm{T}$ cell proliferation, cytokine release, and cytolytic activity of PD-1-positive T cells and promotes tumor cell escaping from host immune attack [18]. Therefore, targeting PD-1/PD-L1 signaling can enhance the capacity of activated $\mathrm{T}$ cells to recognize and kill tumor cells and subsequently, restore the function of host immune surveillance, by the activation of PI3K/Akt and Ras/MEK/Erk signaling pathways (Figure 1) [19, 20]. Given its important role in immunosuppression, the immune checkpoint signaling has been utilized as novel targets for developing antitumor agents.

Nowadays, several anti-PD-1/PD-L1 antibodies have been licensed for treatments of many types of solid tumors in clinical settings, including the NSCLC (for details see Table 1) and many are in developed. Among four of them, nivolumab (Opdivo) [21], pembrolizumab (Keytruda) [16], atezolizumab (Tecentriq, MPDL3280A, RG7446), and durvalumab (Infinzi) [22] have been approved for patients with NSCLC.

Despite the drug blockading the $\mathrm{PD}-1 / \mathrm{PD}-\mathrm{L} 1$ pathways has been shown exciting therapeutic benefits for patients with advanced NSCLC, less than $20 \%$ NSCLC patients could benefit for these novel agents [23-25], suggesting an unmet need for identification of patients in this type of immunotherapy. Indeed, clinical trials have showed that examining of PD-L1 by IHC assays may help in guiding NSCLC patients to choose agents.

In this respect, at least six antibodies are currently used to assay PD-L1 expression. These agents all have a biomarker assay linked to their use. However, only the $22 \mathrm{C} 3$ pharmDx assay has status as a companion diagnostic for use of pembrolizumab. The PDL1 IHC 22C3 pharmDx assay and Ventana PD-L1 (SP142) assay have status as complementary diagnostics for nivolumab and atezolizumab, respectively [26, 27]. 


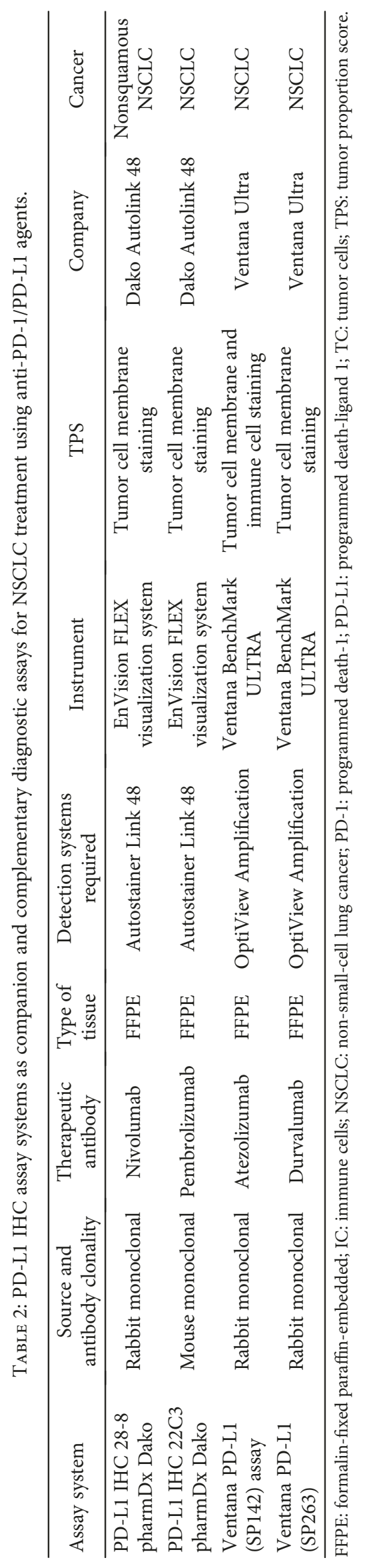




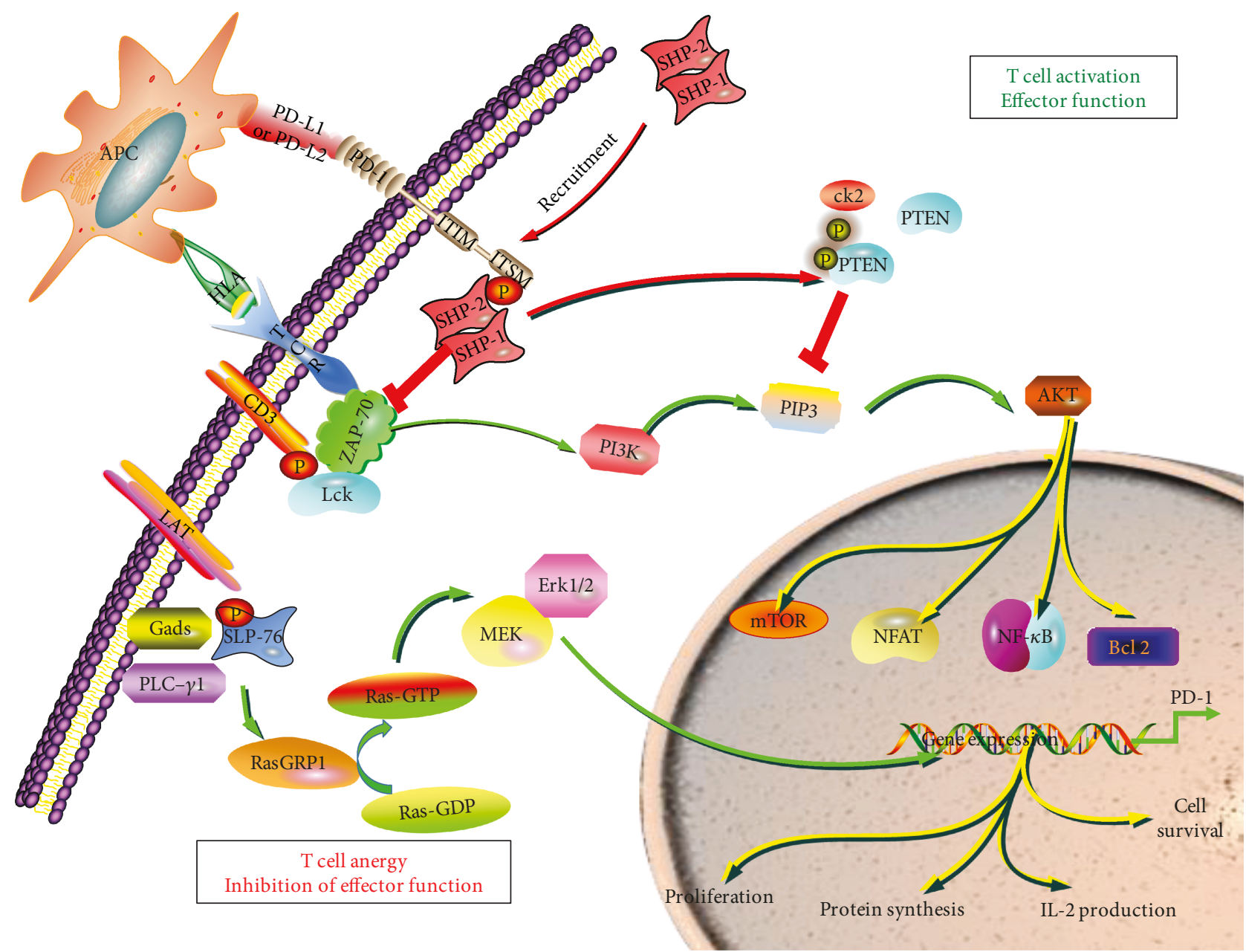

FIGURE 1: Effect of PD-1/PD-L1 signaling on major signaling pathways and reprograming in T cells. Upon the stimulation of antigen, the MHC on the surface of APC could present antigens to the TCR and promote TCR/CD3 chains to phosphorylate, resulting in an activation and recruitment of Lck and Zap-70, which in turn lead to the phosphorylation of tyrosine motifs (ITAM) and initiation of the downstream signaling cascade of TCR. However, in the pathological state, the PD-1 bind to its ligand PD-L1 or PD-L2; the tyrosine phosphatase SHP-2 or SHP-1 can be recruited and bind to the ITSM sequence in the PD-1 cytoplasmic tail. An activation of PD-L/PD-L1 signaling PD-1 mediates the inhibition of the PI3K/Akt and Ras/MEK/Erk signaling pathway, resulting in the inhibition of $\mathrm{T}$ cell proliferation, protein synthesis, survival, and IL-2 production. APC: antigen-presenting cell; HLA: human leukocyte antigen; TCR: T cell receptor.

In 2015, two IHC assays of PD-L1 together with their corresponding agents for NSCLC patients have been authorized for by FDA and/or EMA: one is PD-L1 IHC 22C3 pharmDx assay and the other is 28-8 assay. The Dako 22C3 IHC assay is a companion diagnostic assay for pembrolizumab (Keytruda) (https://www.accessdata.fda. gov/cdrh_docs/pdf15/P150013S006A.pdf), and the 28-8 assay is a complementary diagnostic for nivolumab (Opdivo) (https://www.accessdata.fda.gov/cdrh_docs/pdf15/p150027c .pdf). Similarly, the SP142 PD-L1 IHC assay is developed as the complementary diagnostic with atezolizumab (Tecentriq) (https://www.accessdata.fda.gov/cdrh_docs/pdf16/p16 0002c.pdf), and Ventana SP263 is developed with durvalumab (Imfinzi) (https://www.accessdata.fda.gov/cdrh_docs/ pdf16/p160046c.pdf). Both of SP142 and Ventana SP263 assays are developed by Ventana Medical System for Roche
(Roche, Tucson, AZ, USA). The 78-10 antibody, however, was developed to company with avelumab (Bavencio) [28]. These three assay platforms are developed by Dako Company (Current Agilent, Santa Clara, CA, USA). In addition, a diagnostic IHC assay using E1L3N antibody has also been developed for both the Dako and Ventana platforms [28, 29].

Clinically, PD-L1 IHC 22C3 pharmDx is a qualitative IHC assay for NSCLC tissue in vitro diagnostic and helps to identify NSCLC patients for curing with pembrolizumab, which applies monoclonal mouse anti-PD-L1. The antibody produced by clone $22 \mathrm{C} 3$ was able to recognize and bind to the PD-L1 protein in formalin-fixed, paraffin-embedded (FFPE) NSCLC tissue. The sections are stained with monoclonal mouse anti-PD-L1 or the negative control reagent (NCR) by using an EnVision FLEX visualization system on the Autostainer Link 48 system. The level of PD-L1 protein 
expression was evaluated by the standard of tumor proportion score (TPS). The PD-L1-positive cells for this assay ranged from as low as $1 \%$ to as high as $50 \%$. The specimen should be considered PD-L1-positive if the TPS of viable tumor cells with membrane staining at any intensity was $\geq 1 \%$ (Table 2 ) [30]. A previously treated patient with $\geq 1 \%$ PD-L1-positive cells in tumor might benefit from pembrolizumab as a secondary line therapy, and a previously untreated patient with $\geq 50 \%$ PD-L1-positive cells in tumor might benefit from pembrolizumab as the first-line therapy [26].

PD-L1 IHC 28-8 pharmDx is also an IHC assay for the diagnosis of IHC in clinical trials with nivolumab, by detecting PD-L1 protein in FFPE section of nonsquamous NSCLC and melanoma tissues. The sections are stained with monoclonal rabbit anti-PDL1 or NCR by using an EnVision FLEX visualization system on the Autostainer Link 48 system to visualize the result. $\mathrm{A} \geq 1,5$, and $10 \%$ of $\mathrm{PD}$-L1-positive cells in NSCLC tumors detected by this assay were recommended in various clinical trials [26].

Ventana PD-L1 (SP142) assay is a diagnostic assay complementary to treatment using atezolizumab. It is a qualitative immunohistochemical assay using rabbit monoclonal primary antibody to recognize PD-L1 in paraffin-embedded tissue sections of NSCLC and urothelial carcinoma. In NSCLC, a patient who was detected with a PD-L1 expression $\geq 50 \%$ in tumor cells or $\geq 10 \%$ in immune cells would be eligible for treatment with atezolizumab [26, 29].

Similarly, Ventana PD-L1 (SP263) assay is another PD-L1 protein detecting system developed for NSCLC and other tumor types by the Ventana BenchMark ULTRA [31]. It has been tested as a companion assay for employment of nivolumab, pembrolizumab, and durvalumab. Of note, the SP263 assay could expand the therapeutic range for NSCLC patients. In this regard, identified NSCLC patients can use pembrolizumab and identified nonsquamous NSCLC patients can select nivolumab. For pembrolizumab, patients who were detected $\mathrm{a} \geq 50 \% \mathrm{PD}$-L1-expressing tumor cells in NSCLC tissues could be recommended for a first-line therapy, while those who had a $\geq 1 \%$ PD-L1-positive tumor cells are for a second-line treatment. Noteworthy, $a \geq 1,5$, and $10 \%$ of PD-L1-positive cells in NSCLC tumors detected by SP263 were reported in different clinical trials $[29,31]$. The introduction of PD-L1-based companion and complementary diagnostic assays has greatly increased the efficacy and safety of anti-PD-1/PD-L1 therapy in selected patients, suggesting that the PD-L1 is one of the predictive biomarkers for PD-1/PD-L1 inhibitors in NSCLC.

In order to determine technical equivalences of the above four IHC assays and alterations of antibodies on other staining platforms, comparisons of these PD-L1 IHC assays in NSCLC have been performed by several groups $[32,33]$. The study by Fujimoto et al. suggested an equivalent predictive performance of 28-8, 22C3, and SP263 PD-L1 IHC assays, while the SP142 assay exhibited a lower predictive performance in NSCLC [32]. However, results from the study of Hendry et al. reported that only the 28-8 and 22C3 IHC PD-L1 assays showed a concordant performance and could be used interchangeably in clinical settings [33].

\section{PD-L1 as a Predictive Marker of Response to PD-1/PD-L1 Inhibition in NSCLC Treatment}

A compelling body of evidence has shown that anti-PD-1/ PD-L1 therapy is a promising treatment strategy with unprecedented survival benefits for selected NSCLC patients. Nevertheless, a substantial proportion of patients who received PD-1/PD-L1 inhibitors have little or no benefit. Therefore, it is a need to identify biomarkers for establishment of valid predictors of treatment responses [34]. In this regard, the expression of immunosuppressive molecules including PD-L1, PD-1, and indoleamine 2,3-dioxygenase (IDO), as well as mutational landscape and mutational load [35] and mismatch repair deficiency (MMRD) [36], has been examined as potential predictor of response to anti-PD-1/ PD-L1 treatments. Among them, the expression of PD-L1 in tumors has been employed as the criteria in some of the studies of anti-PD-1/PD-L1 therapy for NSCLC.

Indeed, several clinical trials have reported remarkable results of therapeutic outcome of immune checkpoint blockades in NSCLC patients guided by PD-L1 IHC assays $[11,12]$. Currently, aforementioned four commercial IHCbased bioassays are employed as diagnostic assays for detecting PD-L1 expression of tumors to guide the use of PD-1/ PDL1 inhibitors alone or in combination with other therapeutic agents with different cutoff values in distinct types of lung cancer (for details see Table 2).

Durvalumab (Imfinzi, MEDI4736) is a human IgG1 anti-PD-L1 mAb, which showed a potential to improve progression-free survival in patients with locally advanced, unresectable stage III NSCLC, which met its primary endpoint in the PACIFIC trial for assessing its effects in patients with locally advanced NSCLC after standard chemoradiotherapy [37, 38]. In addition, its potential may be strengthened if the ongoing phase III randomized studies of first line (NCT02453282, MYSTIC trial) and second or subsequent (NCT02352948, ARCTIC trial) lines of therapy demonstrate superiority over the current standard of care [39]. Thus, it was approved for treatment of patients with locally advanced, unresectable stage III NSCLC who do not relapse after platinum-based chemoradiation on Feb. 16, 2018. Its optimal clinical role in managing lung cancer remains unclear, and more robust predictive biomarkers are needed, although the Roche Ventana SP263 has been used as a diagnostic assay for treatment with durvalumab [37, 38]. In addition, the Roche Ventana SP263 assay is used in an ongoing phase III study of durvalumab monotherapy in patients with PD-L1 positivity ( $\geq 25 \%$ tumor cells stained using Roche Ventana SP263) and the durvalumab combined with or without tremelimumab (ARCTIC trial) or either agent versus standard of care (SoC) (such as erlotinib, gemcitabine, and vinorelbine) in patients with PD-L1 tumors [39]. However, AstraZeneca and MedImmune recently reported that the ARCTIC trial did not meet the primary endpoint of improving PFS compared to SoC in NSCLC patients who had a $25 \%$ or more of tumor cells expressing PD-L1 in tumor as determined by the Ventana PD-L1 SP263 assay. The trial is ongoing to assess two additional primary endpoints of overall survival (OS) for durvalumab 
monotherapy and OS for the durvalumab and tremelimumab combination.

Atezolizumab was approved for second-line treatment of patients with advanced NSCLC based on PD-L1 expressing in tumor tissues. In the phase II trial of atezolizumab as first-line or subsequent therapy in advanced NSCLC patients selected with PD-L1 (BIRCH) (NCT02031458), 3914 NSCLC patients were enrolled in accordance with PDL1 expression in tumor-infiltrating immune cells (IC) or tumor cells (TC) and received atezolizumab at a dose of $1200 \mathrm{mg}$ every 3 weeks. The TC PD-L1 positive was defined as TC $3 \geq 50 \%$ or TC $2 \geq 5 \%$ but $<50 \%$, and the IC PD-L1 positive was defined as IC $3 \geq 10 \%$ or IC $2 \geq 5 \%$ but $<10 \%$, as determined by the PD-L1 expression using the SP142 immunohistochemistry assay. This phase II study was divided into three groups: cohort 1 (without chemotherapy advanced NSCLC, 1L), cohort 2 (first-line platinum-containing chemotherapy advanced late NSCLC, 2L), and cohort 3 (at least 2-line chemotherapy advanced late NSCLC, $\geq 3 \mathrm{~L}$ ). The one-year ORR was $22 \%, 19 \%$, and $18 \%$ for cohorts 1,2 , and 3 , respectively; and the ORR in the TC3 or IC3 subgroup for respective cohorts 1,2, and 3 was $31 \%, 26 \%$, and $27 \%$. The OS in cohorts 1, 2, and 3 was 23.5 (26.9 months for TC3 or IC3 patients), 15.5, and 13.2 months, respectively. This study showed that the atezolizumab monotherapy was safe and tolerable for PD-L1-selected advanced NSCLC patients [40].

Of note, these PD-L1 IHC assays showed similarities and differences in clinical trials. In order to better unravel the reliability of these IHC assays in patient selection for antiPD-1/PD-L1 therapy, the Blueprint PD-L1 IHC Assay Comparison Project was founded (AACR, 2015) [17, 31]. In this project, Rimm et al. and Scheel and Schäfer compared five PD-L1 IHC assays (22C3, 28-8, SP142, SP263, and $73-10)[17,31]$. In the clinical phase I trial, they observed that $22 \mathrm{C} 3,28-8$, and SP263 assays were closely aligned on tumor cell staining, whereas the SP142 assay showed fewer stained tumor cells in 19 of 38 samples (50.0\%). Nonetheless, NSCLC tumors were classified using respective criteria of the above 4 assays; 5 of $38(13 \%)$ were determined below the selected cutoffs of all assays. By comparing assays and cutoffs, this data demonstrated that the analytical performance of PD-L1 expression was comparable among 22C3, 28-8, and SP263 assays. A change of assays and cutoffs would lead to "false classification" of PD-L1 states in some patients. Therefore, more data is needed to inform the use of alternative staining assays on PD-L1 cutoff values for different specific therapies [31].

Interestingly, SP142 antibody showed an outlier that has a significantly less mean score of the PD-L1-positive rate in both tumor and immune cells (tumor cells: 1.99; immune cell: 1.62) relative to other three antibodies (tumor cells: 22c3, 2.96; 28-8, 3.26; E1L3N, 3.20; and immune cells: 22c3, $2.15 ; 28-8,2.28$; E1L $3 \mathrm{~N}, 2.28)$ in this study. In addition, the intraclass correlation coefficients (ICCs) of the pathologist score and concordance suggested that pathologists have significant concordance for scores of PD-L1 expression in tumor cells (ranged from ICCs of 0.832 (95\% CI, 0.8200.844 ) to 0.882 ( $95 \% \mathrm{CI}, 0.873-0.891)$ ), but there were poor concordance of ICC immune cells stained with any antibody ranged from $0.172(95 \% \mathrm{CI}, 0.156-0.189)$ to 0.229 (95\% CI, $0.211-0.248)[17,31]$. In the same project, Rimm et al. compared the PD-L1 expression in 90 of stages I-III NSCLC samples using four IHC assays (22C3, 28-8, SP142, and E1L3N) [17]; the result was similar to the above study [31]. Together, these results indicated that 22C3, 28-8, SP263, and E1L3N were better assays for PD-1 expression in clinical settings. Recently, several lines of studies in PD-L1 IHC assays for NSCLC using 22C3 and 28-8 further supported the results of the above two studies [31] [41].

In order to unravel the relationship between PD-L1 expression and NSCLC patient subsets, Igawa et al. accessed the expression of PD-L1 in 229 consecutively resected NSCLC specimens using SP263 immunohistochemical assay and found that the PD-L1 expression was significantly associated with male patients or current smokers [41]. Moreover, a higher PD-L1 expression was determined in squamous NSCLC samples as compared with nonsquamous NSCLC samples (53 and $71 \%$, respectively; $P=0.026$ ) [41]. In addition, the high expression of PD-L1 was associated with a low 5-year survival rate in squamous NSCLC [41]. Aforementioned studies thus suggest that an identification of subtypes of NSCLC is the first step in PD-1/PD-L1 therapy for precise treatment.

In addition, PD-L1 may be also as a biomarker in stage III/IV lung squamous cell carcinoma (SQC). A preliminary study of Chinese patients with stage III/IV lung SQC showed that PD-L1 expression was detected in $61.7 \%(79 / 128)$ of patients. Interestingly, more abundant PD-L1 were observed in samples of smokers over nonsmokers (66.0\% versus $44 \%$, $P=0.042$, resp.). However, a combination of PD-L1 expression and clinicopathologic parameters showed no significant difference in the objective response rate (ORR) for platinumbased chemotherapy between PD-L1-negative and positive patients $(43.3 \%$ versus $36.2 \%, P=0.434 ; 80.0 \%$ versus $78.7 \% P=0.840$, resp.), the overall survival (OS) in PD-L1negative patients was significantly lower than PD-L1positive patients (41.5 versus 19.3 months, $P=0.001$ ) [42]. This study indicated that the PD-L1 was a potential biomarker for anti-PD-1/PD-L1 therapy in smoking-related lung SQC.

Despite an increased number of clinical trials has demonstrated that the PD-L1 is a valuable biomarker for guiding anti-PD-1 and anti-PD-L1 treatments in various cancers [42], the correlation between the PD-L1 expressions determined by an IHC assay could not been reproduced across various studies and tumor subtypes. For instance, the PD-L1 expression was clearly correlated with the clinical response to treatment of nivolumab and pembrolizumab for nonsquamous NSCLC [23, 24], but not correlated with the response in squamous cell NSCLC [23]. Moreover, the PD-L1 expression in immune effector cells of tumor microenvironment is also a potential predictor $[43,44]$. For example, the reservoir of PD-L1-negative TILs provided an immune-privileged microenvironment with a positive impact on survival of patients with resected disease and response to nivolumab in advanced NSCLC patients with intrinsic variability of PDL1 expression in tumors [45]. Currently, there is no standard of PD-L1 IHC assay that has been recommended in a guideline of cancer therapy 
using immune checkpoint blockades. Therefore, there is a need to harmonize the available PD-L1 assays in clinical practice. Nonetheless, the introduction of PD-L1 IHC assays in anti-PD-1/PD-L1 therapy has dramatically increased the therapeutic benefits in NSCLC patients, suggesting an importance of $\mathrm{PD}-1 / \mathrm{PD}-\mathrm{L} 1$ as a predictive biomarker for precision medicine in NSCLC using anti-PD-1/PD-L1 therapy (Table 2).

In addition to the PD-L1 as a testing biomarker for antiPD-1/PD-L1 therapy in NSCLC, several other biomarkers were identified for patient selection in checkpoint blockade therapy. These included tumor mutational load, the "inflamed phenotype" such as TILs and immunoscore, TCR clonality, gene signatures, plasma biomarkers such as cytokines [46], and most recently, the tumor mutation burden (TMB) [47]. For instance, NSCLC patients with high nonsynonymous mutation burden showed more durable clinical benefit to treatment with pembrolizumab with a higher PFS and ORR relative to those with less frequent nonsynonymous mutations [35]. Another example is the serum concentration of IL-8 that is also a surrogate biomarker for predicting response in melanoma and NSCLC patients treated with pivolumab or pembrolizumab alone or nivolumab plus ipilimumab [48]. Of great interest, high TMB is a poor prognostic factor in NSCLC [47], a most recently whole-exome sequencing (WES) study of NSCLC patients treated with PD-1 plus CTLA-4 blockade; however, the study demonstrated that TMB was independent of PD-L1 expression and was the strongest feature associated with improved objective response, durable benefit, and PFS in PD-1 plus CTLA-4 combination of immunotherapy in NSCLC [49]. These findings suggest that high TMB is a predictor of the efficacy of immune, which may be an independently predictive marker for benefits of combination immunotherapy in NSCLC with PD-L1 and CTLA-4 blockade in future clinical trials $[47,49]$.

\section{PD-1/PD-L1 Signaling Pathway as a Therapeutic Target for NSCLC}

Targeting the PD-1/PD-L1 signaling pathway has recently become a promising therapeutic strategy in cancer therapy. Such a strategy is through a mechanism by preventing the escape of tumor cells from the immune surveillance and restoring the function of the host's immune system to attack the tumor cells (Tables 1 and 3) [11].

The encouraging clinical safety and effects of anti-PD-1/ PD-L1 antibodies in both clinical trials and settings have led a fast development of inhibitors of PD-1 and PD-L1/2 for treatments of solid tumors, including the NSCLC. In this regard, nivolumab was firstly assessed for its safety and clinical activity in 39 patients with intractable solid tumors (including advanced metastatic NSCLC, melanoma, colorectal cancer (CRC), castrate-resistant prostate cancer, or renal cell carcinoma (RCC)) [50]. Subsequently, a dose-increasing trial of multiple doses of nivolumab was conducted in 296 patients with advanced malignancies, including 122 patients with NSCLC (with or without EGFR or KRAS mutation, squamous or nonsquamous type, $\mathrm{PD}-1$ expression positive or negative) [51]. This objective response rates (ORRs) were $28 \%$ (26 of 94 melanoma patients), 27\% (9 of 33 RCC patients), and $18 \%$ (14 of 76 NSCLC patients) (NCT00730639) [51]. Responses were observed for at least 1 year in the great mass of patients. Furthermore, preliminary data indicated that the objective response was correlated with PD-L1 expression of cancer tissue as determined by immunohistochemical analysis [51]. Meanwhile, the phase I study using another anti-PD-L1 monoclonal antibody (BMS936559) was demonstrated to give rise to durable tumor regression with the ORR ranging from $6 \%$ to $17 \%$ in advanced cancers, including 75 with non-small-cell lung cancer (NCT00729664) [52]. On the basis of these phase I results, several randomized, open-label, and international phase 3 trials were initiated [23, 53, 54]. One study was designed to compare nivolumab with docetaxel in advanced nonsquamous NSCLC [53]. In this study, the patient group treated with nivolumab resulted in advancing median overall survival (OS) compared to docetaxel (OS 12.2 versus 9.4 months); the OS at one year was $51 \%(95 \%$ CI, 45 to 56 ) in patients treated with nivolumab versus $39 \%$ (95\% CI, 33 to 45 ) in the docetaxel group (NCT01673867) [53]. At the same time, the phase III study by Brahmer et al. followed the same design and showed an increased OS in the nivolumab group compared to the docetaxel group (9.2 versus 6.0 months, resp.) (NCT01642004). Unexpectedly, the expression of PD-L1 in tumor determined at three different expression cutoff values $(1,5$, and $10 \%)$ showed neither prognostic nor predictive treatment benefit in this study [23]. However, a randomized phase II study in investigation of the efficacy and safety of atezolizumab comparing with docetaxel in previous platinum-treated NSCLC patients (who were PD-L1-positive as determined by SP142 antibody IHC assay) showed that atezolizumab resulted in an enhanced PFS (9.7 versus 3.9 months) and OS compared to docetaxel (12.2 versus 9.4 months) for patients with high levels of PD-L1 expression (NCT01903993) [54].

The promising results of clinical trials led FDA to approve nivolumab for treatment of intractable metastatic squamous NSCLC in 2015. Almost at the same time, another anti-PD-1 antibody, pembrolizumab, was licensed for treatment of PD-L1-positive advanced NSCLC by FDA/EMA, based on a mass of clinical trials exhibited that pembrolizumab was safe and effective for metastatic NSCLC (mNSCLC) $[14,15]$. Moreover, the cutoff $>50 \%$ PD-1-positive patients exhibited a better benefit from pembrolizumab therapy [14]. It therefore was approved for first-line treatment of patients with mNSCLC whose tumors have high PD-L1 expression (tumor proportion score (TPS) $\geq 50 \%$ ) and wild type of EGFR or ALK genes [55]. This approval expanded the indication of pembrolizumab as a second-line treatment of lung cancer. Indeed, meta-analysis in randomized clinical trials of anti-PD-1/PD-L1 therapy (atezolizumab, pembrolizumab, and nivolumab) demonstrated the efficacy and safety and could dramatically improve the PFS and OS compared with docetaxel for patients with previously treated NSCLC $[56,57]$ and enhance the PFS and OS compared to EGFRTKI alone for NSCLC patients with EGFR wild type [56]. Of note, as far as PFS was a concern, an anti-PD-1/PD-L1 therapy was second only to EGFR-TKI therapy for patient 


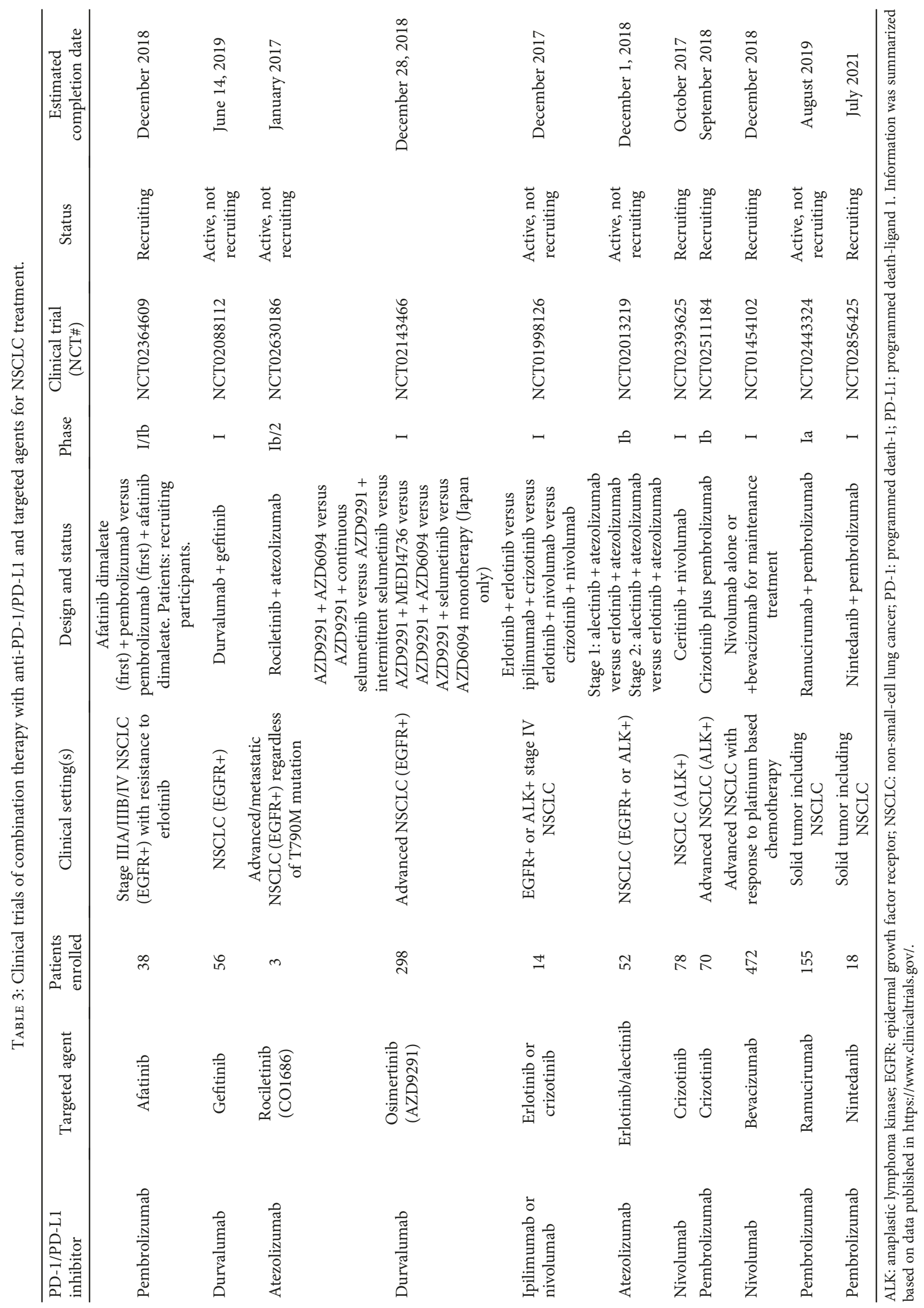


with EGFR mutation [56]. These clinical trials clearly demonstrated that inhibition of the PD-1/PD-L1 pathway could clinically improve the ORR, OS, and PFS compared with single-arm chemotherapy alone in nonsquamous and squamous NSCLCs. In addition, these data also showed that PD-1 or PD-L1 could serve as a biomarker to improve the benefit of anti-PD-1/PD-L1 therapy for NSCLC patients.

\section{Anti-PD-1/PD-L1-Based Combinatory Therapies for NSCLC Treatment}

Owing to the lack of a definite biomarker for the selection of patients who likely benefit from checkpoint inhibitor-based monotherapy, anti-PD-1/PD-L1-based combinatory therapies were designed. In addition, the primary or acquired resistance to immune checkpoint inhibitors and/or conventional therapies is inevitable in most cases of NSCLC. Therefore, combinatory therapies designed to reenergize the immune system with complementary/synergetic mechanisms were introduced as an alternative strategy for NSCLC treatment in clinical settings. These studies are on the basis of anti-PD-1/PD-L1 agents with other immunotherapies (such as CTLA-4 blockade), chemotherapy, radiotherapy, and targeted therapy [44, 49, 58-62].

A compelling body of studies has demonstrated that the PD-L1 expression was associated with EGFR mutation, ALK rearrangements, or KRAS mutation in NSCLC [21, 63-67]. These studies evidenced that oncogene drivers could enhance immune escape of tumor cells by upregulating PD-L1 expression in NSCLC. For example, the PD-L1 expression was linked to KRAS mutations and was significantly associated with EGFR mutations [68]. Moreover, an activation of the oncogenic EGFR signaling pathway could enhance PD-L1 expression and suppress antitumor immunity [69]. These studies suggest that a combination of PD-1/PD-L1 blockades with EGFR-TKIs may enhance the outcome of NSCLC treatment. In this respect, a combination of nivolumab with erlotinib showed an increased OS, ORR, and PFS in NSCLC patients with acquired resistance to erlotinib [70]. The combinatory therapy of durvalumab and gefitinib (NCT02088112) [71] and durvalumab (NCT0214346) [72] also showed an increased ORR in NSCLC patients with EGFR mutation. However, the combination of durvalumab and osimertinib (AZD9291) has been held due to the unacceptably high rate of pneumonitis.

In addition, PD-L1-positive patients exhibited more sensitive to gefitinib or erlotinib than PD-L1-negative patients in terms of TTP and OS [68]. Interestingly, an acquired resistance to gefitinib could enhance the PD-L1 expression and $M E T$ positivity in EGFR-mutant NSCLC [73]. Similarly, NSCLC patients harboring mutant KRAS treated with immune checkpoint inhibitors (ICIs) had prolonged OS relative to those containing wild-type KRAS [65]. These results implied that high mutational rates of EGFR or KRAS mutations enhanced immunogenicity and could serve as potential biomarkers for anti-PD-1/PD-L1 therapy $[65,69]$. These findings were supported by recent whole-exome sequencing (WES) studies in NSCLC, in which patients with higher TMB benefited more from a combination immunotherapy in NSCLC $[47,49]$.

Indeed, NSCLC patients with acquired resistance of EGFR-TKIs and EGFR mutation but not T790M were reported to benefit more from nivolumab than those with T790M mutation [74]. In this study, 25 patients with EGFR mutation-positive NSCLC (cohort A) and 60 patients with acquired EGFR-TKI resistance who were included in whole-exome sequencing (cohort B) were treated with nivolumab. The results displayed that the median PFS of patients with the T790M-negative PD-L1 expression level of $\geq 1 \%$ and those who had a T790M-positive PD-L1 expression level of $<1 \%$ were 2.1 and 1.3 months, respectively. Generally, the PD-L1 expression of $\geq 10 \%$ or $\geq 50 \%$ was associated with PFS in T790M-negative patients. These results warrant further investigation for prospective outcomes of clinical trials [74]. Further studies on the relationship between driver oncogene mutations (EGFR, ALK, KRAS, MET, and ROS1) and immune-related biomarkers (PD-1, PD-L1, CTLA-4, and CD8) in NSCLC demonstrated that the PD-L1 expression of $26 \%$ of SCC and $76 \%$ of adenocarcinoma samples was overlapped with driver oncogenes [75], but a TPS of $\mathrm{PD}-\mathrm{L} 1 \geq 50 \%$ was rarely overlapped with driver oncogenes [76]. Interestingly, frequent PD-L1/CD8-double positive TILs were observed in TPS [75], and high density of CD8-positive TIL and nonsynonymous mutation burden were found in nivolumab responders [74]. To date, several clinical trials of a combination of anti-PD-1/PD-L1 agents (atezolizumab, pembrolizumab, and nivolumab) with EGFRTKIs (gefitinib and erlotinib) for NSCLC treatment are undergoing (Table 3), and these are identified as a major breakthrough in advanced or previously treated advanced NSCLC. It is worthy to note that the relationship of PD-L1 expression and EGFR oncogenic mutations is controversial between different studies. Both low and high EGFR mutations with PD-L1 expression were reported [77]. In addition, treatments of chemoradiation and TKIs might also alter the PD-L1 expression.

Chemotherapy, particularly the platinum-based doublet chemotherapy (PT-DC) is the first-line treatment for patients with advanced NSCLC. Interestingly, an increased number of evidences unraveled that chemotherapy has an impact on immune microenvironment of tumors, which in turn enhances its antitumor, through mechanisms including reduction of T-regulatory cell activity, selective depletion of myeloid-derived suppressor cells (MDSCs), induction of PD-L2 expression, and the maturation of APCs [78, 79]. Therefore, a combination of anti-PD-1/PD-L1 therapy with standard chemotherapy may result in a synergistic antitumor activity for NSCLC treatment. Indeed, a growing body of clinical studies has shown encouraging outcomes for combination therapies of each of three approved anti-PD-1/PD-L1 agents, pembrolizumab, nivolumab, and atezolizumab with chemotherapy in NSCLC. The result of phase II randomized trial of pembrolizumab plus chemotherapy (carboplatin and pemetrexed) showed a $55 \%$ of ORR, which was significantly increased in comparison with the $29 \%$ ORR in treatment with first-line chemotherapy alone for advanced nonsquamous NSCLC [80]. Similarly, results from the CheckMate 
012 trial also showed a range of $33 \%-47 \%$ of ORR in first-line treatment of combinatory therapy of nivolumab and chemotherapy for advanced NSCLC [81]. Similarly, the combination of atezolizumab with platinum-based doublet chemotherapy resulted in a promising activity (ORRs: $60 \%-75 \%$ ) with no unexpected toxicities as first-line therapy for locally advanced or mNSCLC (NCT01633970) [82]. Of note, the sample sizes of the above studies were small and lack of data of the improvement of PFS and OS. In addition, the adverse effect of the combination therapy of immune checkpoint inhibitors with chemotherapy was a safety concern. Therefore, a precise dosing regimen and ingenious strategy design are of importance to exert maximum antitumor activity with tolerable toxicity. Nevertheless, the robust antitumor activity and reliable safety profile of immune checkpoint inhibitor-combined chemotherapy motivated more phase III studies to investigate the efficacy and safety of a regimen including an immune checkpoint blockade for the first-line treatment of advanced NSCLC, and more randomized, double-blind, large cohort, phase III studies are planned or undergoing. Encouragingly, the study of Keynote-189 (NCT02578680) phase III trial recently reported that the combination of pembrolizumab and standard chemotherapy of pemetrexed and a platinum-based drug yielded a significantly longer OS and PFS over chemotherapy alone in metastatic NSCLC [83]; in addition, the IMpower150 (NCT02366143) phase III trial also showed a remarkable improvement of the OS and PFS of NSCLC patients treated with a combination of atezolizumab and bevacizumab plus carboplatin and paclitaxel significantly, compared to bevacizumab plus carboplatin and paclitaxel alone [84].

Similar to chemotherapy, radiotherapy is another common nonsurgical treatment for NSCLC. In addition to its ability to directly kill tumor cells, radiation is also able to trigger local immune responses and subsequently render the tumor microenvironment to recruit effective $\mathrm{T}$ cells [85]. However, the radiation-triggered immune response is far to generate a systemic antitumor immunity. Intriguingly, immune checkpoint inhibitors exhibit a capacity to enhance the radiotherapy-triggered local immune response to systemic antitumor effects, that is, abscopal effects [79, 86, 87]. Indeed, substantial data have shown that the addition of immune checkpoint inhibitor increased the effect of either radiotherapy or immunotherapy alone [88, 89]. In this respect, the ablative and highly targeted doses of stereotactic ablative radiotherapy (SABR) in combination with anti-PD1/PD-L1 therapy have spurred an increased interest, since SABR can induce more robust immune response and reduce surrounding normal tissue toxicity than conventionally fractionated radiotherapy [90]. Of importance, an administration of PD-1/PD-L1 inhibitor before or during the radiotherapy is a more reasonable approach to bring a long-term antitumor effect than the immune checkpoint inhibitors which are given after the radiotherapy, as the concurrent chemoradiation therapy (CCRT) may induce the expression of PD-L1 [91, 92]. However, recent PACIFIC trial showed remarkable efficacy of durvalumab when it was administered following the completion of CCRT $[44,93]$, suggesting that further trials are required to optimize therapeutic regimens in combination of immune checkpoint blockades and CCRT. Indeed, several lines of clinical studies of anti-PD-1/PD-L1 therapy in combination with radiotherapy in the treatment of NSCLC are currently undergoing [94].

It has been demonstrated that angiogenic factors are immunosuppressive, implying that a combination of immune checkpoint blockade with antiangiogenic agents may exhibit synergistic antitumor activity in NSCLC treatments. Indeed, primary data from clinical trials in combination therapy with immune checkpoint blockade (nivolumab and pembrolizumab) and antiangiogenic agents (bevacizumab, ramucirumab, and nintedanib) show promising results for NSCLC [60]. For example, the phase I trial (NCT01454102) evaluated the efficacy and safety of combination maintenance treatment of nivolumab with bevacizumab in advanced NSCLC patients with response to first-line platinum-based chemotherapy. Preliminary results reported an acceptable toxicity profile of combination treatment with median PFS of 37.1 weeks in nonsquamous patients, but the nivolumab monotherapy showed the respective median PFS of 21.4 and 16 weeks for nonsquamous and squamous NSCLC patients [38]. Several other trials exploring a combination of immune checkpoint blockade and antiangiogenic agents are underway, including the combination of ramucirumab with pembrolizumab (NCT02443324) in patients with advanced solid tumors including NSCLC [95], pembrolizumab with nintedanib (NCT02856425) in advanced NSCLC [96], and bevacizumab plus chemotherapy with atezolizumab (NCT02366143) or pembrolizumab (NCT02039674) in the first-line setting for NSCLC treatment (Table 3) [60]. Remarkably, the phase III IMpower150 (NCT02366143) study has showed that the combination of atezolizumab and bevacizumab plus carboplatin and paclitaxel significantly improved the OS and PFS in NSCLC patients, compared to bevacizumab plus carboplatin and paclitaxel alone [84].

Indoleamine 2,3-dioxygenase (IDO) is a normal endogenous mechanism of acquired peripheral immune tolerance in vivo and is highly expressed in several cancer types and usually associated with poor prognosis. Trials evaluated anti-PD-1/PD-L1 or CTLA-4 antibodies (nivolumab, pembrolizumab, atezolizumab, and durvalumab) in combination with indoximod, BMS-986205, or epacadostat; inhibitors of IDO1 are underway (NCT02327078, NCT03085914, NCT02178722, NCT02862457, NCT02298153, NCT02318277, and NCT02658890) [97]. The phase I trial examined the combination of epacadostat with pembrolizumab in NSCLC patients exhibited a disease control rate of $58 \%$, with a good safety profile, regardless of PD-L1 status [98, 99].

Apart from combinatory therapies with chemo-, radio-, or targeted therapy, the anti-PD-1/PD-L1 therapy combined with other immunotherapies also is an attractive approach for NSCLC treatment [100-102]. For example, both PD-1/ PD-L1 and CTLA-4 checkpoint inhibitors are capable of enhancing antitumor $\mathrm{T}$ cell activity with different complementary mechanisms. It is therefore an anti-PD-1/PD-L1 


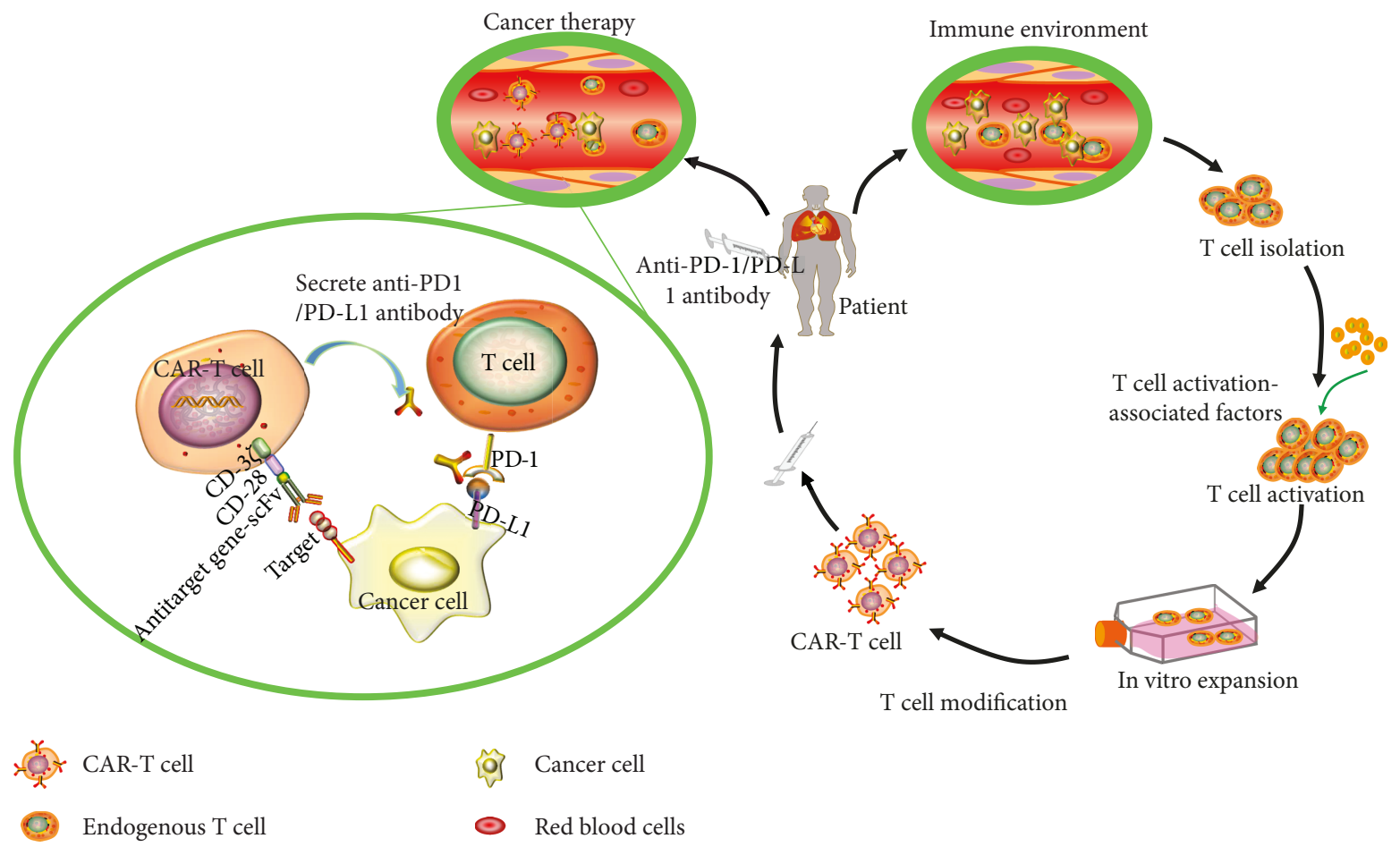

FIgURe 2: Illustrative image describing anti-PD-1/PD-L1 therapy combined with CAR-T cells. T cells are obtained and isolated from the patients. These $\mathrm{T}$ cells are transformed with chimeric antigen receptor (CAR) gene by lentiviruses. The CAR-T cells are expanded in vitro and finally infused back to patients. After the CAR-T cells transferred into patients, the CAR-T cells are able to recognize target gene-positive tumor cells. Meanwhile, CAR-T cells can secrete anti-PD-1/PD-L1 antibody and combine with foreign anti-PD-1/PD-L1 antibody to recognize and kill the tumor cells.

therapy in combination with the anti-CTLA-4 agent may be a potential to improve the antitumor responses of each agent alone. However, clinical studies assessed the combination of durvalumab (anti-PD-L1) and tremelimumab (anti-CTLA4) or pembrolizumab (anti-PD-L1) and ipilimumab (antiCTLA-4) for treatment of NSCLC yielded no better ORR but increased AEs [38, 103, 104], despite encouraged response to the combination of nivolumab and ipilimumab was observed in NSCLC patients with high PD-L1 expression $[37,38]$. The results from the undergoing phase III CheckMate 227 trial (NCT02477826) may offer the more informative data on the combinatory therapy of nivolumab plus ipilimumab in first-line treatment for advanced NSCLC [100].

Interestingly, chimeric antigen receptor (CAR) $\mathrm{T}$ cell (CAR-T) therapy has demonstrated a promising clinical effect in broad of malignancies including chronic lymphoid leukaemia and lymphomas, despite it still faces a series of challenges in treatments of solid tumors. Encouragingly, recent studies unraveled that $\mathrm{PD}-1 / \mathrm{PD}-\mathrm{L} 1$ inhibitors could increase the efficiency of $\mathrm{T}$ cell-based immunotherapy [105]. A previous study found that an antigen-specific stimulation of PD-L1(+) Her-2+ tumor cells could significantly increase the PD-1 expression in CAR + CD8 $+\mathrm{T}$ cells in a syngeneic mouse model, implying that PD-1 blocking antibody could potently increase CAR-T therapy [106]. Indeed, the PD-1-based inhibitory chimeric antigen receptors (iCARs) exhibited an ability to selectively suppress the function of endogenous T cell receptor (TCR) or CAR-T cells. Therefore, blockading PD-1 signaling in combination with CAR-T cell therapy may potentiate the therapeutic efficacy by overcoming the PD-L1+ tumor-mediated immunosuppressive effect. This notion was supported by a recent study in the effect of PD-L1 expression of tumor cells on CAR-T function [102]. Results from this study showed that the PD-L1 expression of tumor cells suppressed the $4-1 \mathrm{BB} \zeta$ CAR-T cell function of tumor clearance in a xenograft model, and a disruption of PD-1 by CRISPR/Cas9-mediated gene editing within CAR-T cells led an augmented CAR-T cell antitumor efficacy. This study thus indicates a promise of CRISPR/Cas9-mediated PD-1 disruption of CAR-T cells for enhancing immunotherapeutic efficacy of CAR-T therapy, which also suggests that the precision genome editing of CAR-T and PD-1 is the next generation of cell therapies (Figure 2) [102].

\section{The Challenge of Resistance to Anti-PD-1/PD-L1 Therapy}

Immune checkpoint blockades have displayed a great potential in cancer therapy, which exhibited a remarkable efficacy compared to conventional treatment for advanced NSCLC $[11,107]$. To date, several monoclonal antibodies (mAbs) to PD-1/PD-L1 or CTLA-4 are approved by FDA for cancer treatments. However, not all patients always benefit from these agents [14], and the data of preclinical and clinical trials 
demonstrated that only $20-50 \%$ patients benefited from antiPD-1/PD-L1 therapy for various cancer types [14, 51, 52]. Equally important, the development of resistance to antiPD-1/PD-L1 immunotherapy further leads to the failure and poor prognosis in advanced NSCLC patients receiving anti-PD-1/PD-L1 treatment. However, the mechanism underling the resistance is not fully understood. In addition, little is known about the contributions of exceeding the PD-L1-positive level, the potential antigen load or mutational load in the tumor and the genetic determinants to efficacy, and resistance to anti-PD-1/PD-L1 therapy [35].

There are several lines of studies in investigating mechanisms of therapeutic resistance to anti-PD-1/PD-L1 agents. A study by Koyama et al. found that an upregulation of $\mathrm{T}$ cell immunoglobulin-3 (TIM-3) in T cells could increase the adaptive resistance to therapeutic PD-1 blockade in fully immunocompetent mouse models of lung adenocarcinoma. Conversely, the sensitivity of anti-PD-1 therapeutic blockade could be restored in these mouse models after addition of anti-TIM-3 antibodies [108]. These results suggested that a strategy by downregulating TIM-3 might enhance the sensitivity of cancer cells to therapeutic PD-1 blockade. More recently, Anagnostou et al. unraveled that the evolving landscape of tumor neoantigens in NSCLC patients who were treated with immune checkpoint inhibitors (ICIs) initially respond and emerge the acquired resistance [43]. In resistant tumor clones, the authors found that 7 to 18 putative mutation-associated neoantigens were lost and the loss of neoantigens occurred either by an elimination of tumor subclones or by a deletion of truncal chromosome regions [43]. Intriguingly, these new mutations did not encode new antigens, suggesting that an expansion of the breadth of new antigenic reactivity could reduce the development of acquired resistance [43]. In addition, an acquired resistance to EGFRTKIs or cytotoxic drugs (cisplatin or vinorelbine) could induce a downregulation of E-cadherin and alter PD-L1 expression in lung cancer cells [109]. These studies suggest a necessity to investigate the mechanism of acquired resistances to immune checkpoint blockades.

\section{Conclusion}

Conventional cancer treatments, including surgical resection, chemotherapy, and/or radiotherapy, have shown modest progress in NSCLC survival over the past two decades. The introduction of targeting agents, such as EGFR-TKI or ALK inhibitor, further offered significant improvements in NSCLC survival carrying an EGFR mutation or ALK rearrangement. To date, there are four anti-PD-1/PD-L1 agents including pembrolizumab (Keytruda), nivolumab (Opdivo), atezolizumab (Tecentriq), and durvalumab (Imfinzi) have been licensed for serving as first- or second-line NSCLC therapy. And other agents, such as MEDI0680, PDR001, REGN2810, BMS-936559, and avelumab (Bavencio) have been entered in preclinical or clinical trials for NSCLC treatment. Therefore, we expect that these agents can improve clinical efficacy and are approved for NSCLC therapy in practice. Indeed, a recent meta-analysis of five trials demonstrated that nivolumab and pembrolizumab were correlated with a significant increase of ORR as compared to atezolizumab, while nivolumab was found to associate with a lower incidence of G3-5 AEs in comparison with other anti-PD1/PD-L1 agents for NSCLC treatment [110].

However, there are grave challenges in anti-PD-1/PD-L1 therapies for NSCLC patients. These include how to identify patients who may benefit from the therapy and minimize the development of acquired resistance to the therapy. Therefore, with an ultimate goal toward the improvement of the therapeutic efficacy and reduction of the adverse effects of antiPD-1/PD-L1 blockades, continuing efforts are required to identify novel predictive biomarkers for patient selection by taking advantage of the rapid development of computational models and high-throughput sequencing techniques for effective and personalized immunotherapy. In addition, with our deeper understanding of mechanisms of immune escape and its role in the biological behaviors of NSCLC, a combinatory approach on the basis of anti-PD-1/PD-L1 therapy, such as a combination with chemotherapy, targeted therapy, radiotherapy, and other immunotherapies, may establish landmarks for treatment of NSCLC. For instance, in NSCLC patients with acquired resistance to anti-PD-1/PD-L1 immunotherapies and EGFR or $A L K$ mutations, particularly for patients with high tumor burden or rapid disease progression, a combination of anti-PD-1/PD-L1 therapy and targeted therapy may be an option. To this end, it is important to comprehend mechanisms of the resistance to anti-PD-1/ PD-L1 agents and identify patients who may potentially benefit from therapeutic schedule. In this respect, the discovery of novel biomarkers and/or development of precise companion diagnostic assays become critical for patient selection. Currently, however, the expression of PD-L1 is only the tip of the iceberg in the predictive index of anti-PD-1/PD-L1 therapy; it is necessary to combine the multiple indexes to make the best predictive ability, indicating an importance of personalized biomarkers in guiding anti-PD-1/PD-L1 immune checkpoint blockade therapy for NSCLC.

\section{Abbreviations}

$\begin{array}{ll}\text { ADC: } & \text { Adenocarcinoma } \\ \text { AEs: } & \text { Adverse events } \\ \text { AKL: } & \text { Anaplastic lymphoma kinase } \\ \text { APC: } & \text { Antigen-presenting cell } \\ \text { BATF: } & \text { Basic leucine transcription factor, ATF-like } \\ \text { BCR: } & \text { B cell receptor } \\ \text { CAR: } & \text { Chimeric antigen receptor } \\ \text { CCRT: } & \text { Concurrent chemoradiation therapy } \\ \text { CK2: } & \text { Casein kinase } 2 \\ \text { CRC: } & \text { Colorectal cancer } \\ \text { CTLA-4: } & \text { Cytotoxic T-lymphocyte-associated antigen } 4 \\ \text { DAG: } & \text { Diacylglycerol } \\ \text { DC: } & \text { Dendritic cells } \\ \text { E2: } & \text { Estrogen 2 } \\ \text { EGFR: } & \text { Epidermal growth factor receptor } \\ \text { EGFR-TKIs: } & \text { Epidermal growth factor receptor tyrosine } \\ & \text { kinase inhibitors } \\ \text { ERK: } & \text { Extracellular signal-regulated kinase }\end{array}$




$\begin{array}{ll}\text { IC: } & \text { Immune cells } \\ \text { iCARs: } & \text { Inhibitory chimeric antigen receptors } \\ \text { ICIs: } & \text { Immune checkpoint inhibitors } \\ \text { ITIM: } & \text { Immunoreceptor tyrosine-based inhibitory } \\ & \text { motif } \\ \text { ITSM: } & \text { Immunoreceptor tyrosine-based switch motif } \\ \text { GM-CSF: } & \text { Granulocyte-macrophage colony-stimulating } \\ & \text { factor } \\ \text { MEK: } & \text { Mitogen-activated and extracellular signal- } \\ & \text { regulated kinase } \\ \text { NK: } & \text { Natural killer cell } \\ \text { MSLN: } & \text { Mesothelin } \\ \text { NSCLC: } & \text { Non-small-cell lung cancer } \\ \text { ORR: } & \text { Overall response rate } \\ \text { ORRs: } & \text { Objective response rates } \\ \text { OS: } & \text { Overall survival } \\ \text { PD-1: } & \text { Programmed death-1 } \\ \text { PD-L1: } & \text { Programmed death-ligand 1 } \\ \text { PFS: } & \text { Progression-free survival } \\ \text { PLC- } \gamma 1: & \text { Phospholipase C gamma 1 } \\ \text { PI3K: } & \text { Phosphatidylinositol 3-kinase } \\ \text { RCC: } & \text { Renal cell carcinoma } \\ \text { RFS: } & \text { Recurrence-free survival } \\ \text { PTEN: } & \text { Phosphatase and tensin homolog } \\ \text { scFv: } & \text { Single chain variable fragment } \\ \text { SHP-2: } & \text { Src homology 2-domain-containing tyrosine } \\ & \text { phosphatase 2 } \\ \text { SHP-1: } & \text { Src homology region 2 domain-containing } \\ & \text { phosphatase-1 } \\ \text { TC: } & \text { Tumor cells } \\ \text { TCR: } & \text { T cell receptor } \\ \text { TIM-3: } & \text { T cell immunoglobulin mucin-3 } \\ \text { TMB: } & \text { Tumor mutation burden } \\ \text { TTP: } & \text { Time to progression. } \\ & \end{array}$

\section{Conflicts of Interest}

The authors declare no conflict of interest.

\section{Authors' Contributions}

Hongshu Sui, Ningxia Ma, Ying Wang, and Yanping Su collected references and wrote the draft; Hui Li collected information. Hui Li, Xiaoming Liu, and Jiali Yang draw figures. Yanping Su and Jiali Yang critically revised the manuscript. All authors read and approved the final version of the manuscript. Hongshu Sui and Ningxia Ma contributed equally to this work.

\section{Acknowledgments}

This work was supported by a grant from the National Natural Science Foundation of China (no. 81670004), a grant from the Ningxia Medical University (XY2017172), a grant from Association of Science and Technology of Ningxia to Jiali Yang, and a grant from Doctoral Scientific Startup Foundation of Taishan Medical University.

\section{References}

[1] R. L. Siegel, K. D. Miller, and A. Jemal, "Cancer statistics, 2017," CA: A Cancer Journal for Clinicians, vol. 67, no. 1, pp. 7-30, 2017.

[2] S. Ramalingam and C. Belani, "Systemic chemotherapy for advanced non-small cell lung cancer: recent advances and future directions," The Oncologist, vol. 13, Supplement 1, pp. 5-13, 2008.

[3] T. Hida, H. Nokihara, M. Kondo et al., "Alectinib versus crizotinib in patients with $A L K$-positive non-small-cell lung cancer (J-ALEX): an open-label, randomised phase 3 trial," The Lancet, vol. 390, no. 10089, pp. 29-39, 2017.

[4] S. Peters, D. R. Camidge, A. T. Shaw et al., "Alectinib versus crizotinib in untreated $A L K$-positive non-small-cell lung cancer," The New England Journal of Medicine, vol. 377, no. 9, pp. 829-838, 2017.

[5] K. Kiura, F. Imamura, H. Kagamu et al., "Phase 3 study of ceritinib vs chemotherapy in $A L K$-rearranged NSCLC patients previously treated with chemotherapy and crizotinib (ASCEND-5): Japanese subset," Japanese Journal of Clinical Oncology, vol. 48, no. 4, pp. 367-375, 2018.

[6] R. Califano, A. Greystoke, R. Lal, J. Thompson, and S. Popat, "Management of ceritinib therapy and adverse events in patients with $A L K$-rearranged non-small cell lung cancer," Lung Cancer, vol. 111, pp. 51-58, 2017.

[7] S. A. Noonan and D. R. Camidge, "PROFILE 1014: lessons for the new era of lung cancer clinical research," Translational Lung Cancer Research, vol. 4, no. 5, pp. 642-648, 2015.

[8] S. Novello, J. Mazières, I. J. Oh et al., "Alectinib versus chemotherapy in crizotinib-pretreated anaplastic lymphoma kinase $(A L K)$-positive non-small-cell lung cancer: results from the phase III ALUR study," Annals of Oncology, vol. 29, no. 6, pp. 1409-1416, 2018.

[9] D. W. Y. Tsui, M. Murtaza, A. S. C. Wong et al., "Dynamics of multiple resistance mechanisms in plasma DNA during EGFR-targeted therapies in non-small cell lung cancer," EMBO Molecular Medicine, vol. 10, no. 6, article e7945, 2018.

[10] F. Han, J. He, F. Li et al., "Emerging roles of microRNAs in EGFR-targeted therapies for lung cancer," BioMed Research International, vol. 2015, Article ID 672759, 10 pages, 2015.

[11] J. Yang, J. Chen, J. Wei, X. Liu, and W. C. Cho, "Immune checkpoint blockade as a potential therapeutic target in non-small cell lung cancer," Expert Opinion on Biological Therapy, vol. 16, no. 10, pp. 1209-1223, 2016.

[12] B. Xia and R. S. Herbst, "Immune checkpoint therapy for non-small-cell lung cancer: an update," Immunotherapy, vol. 8, no. 3, pp. 279-298, 2016.

[13] T. O. Dang, A. Ogunniyi, M. S. Barbee, and A. Drilon, "Pembrolizumab for the treatment of PD-L1 positive advanced or metastatic non-small cell lung cancer," Expert Review of Anticancer Therapy, vol. 16, no. 1, pp. 13-20, 2016.

[14] E. B. Garon, N. A. Rizvi, R. Hui et al., "Pembrolizumab for the treatment of non-small-cell lung cancer," The New England Journal of Medicine, vol. 372, no. 21, pp. 2018-2028, 2015.

[15] S. H. Lim, J. M. Sun, S. H. Lee, J. S. Ahn, K. Park, and M. J. Ahn, "Pembrolizumab for the treatment of non-small cell lung cancer," Expert Opinion on Biological Therapy, vol. 16, no. 3, pp. 397-406, 2016. 
[16] J. Sul, G. M. Blumenthal, X. Jiang, K. He, P. Keegan, and R. Pazdur, "FDA approval summary: pembrolizumab for the treatment of patients with metastatic non-small cell lung cancer whose tumors express programmed death-ligand 1," The Oncologist, vol. 21, no. 5, pp. 643-650, 2016.

[17] D. L. Rimm, G. Han, J. M. Taube et al., "A prospective, multiinstitutional, pathologist-based assessment of 4 immunohistochemistry assays for PD-L1 expression in non-small cell lung cancer," JAMA Oncology, vol. 3, no. 8, pp. 1051-1058, 2017.

[18] M. E. Keir, S. C. Liang, I. Guleria et al., "Tissue expression of PD-L1 mediates peripheral T cell tolerance," The Journal of Experimental Medicine, vol. 203, no. 4, pp. 883-895, 2006.

[19] X. Gong, X. Li, T. Jiang et al., "Combined radiotherapy and anti-PD-L1 antibody synergistically enhances antitumor effect in non-small cell lung cancer," Journal of Thoracic Oncology, vol. 12, no. 7, pp. 1085-1097, 2017.

[20] R. Okita, A. Maeda, K. Shimizu, Y. Nojima, S. Saisho, and M. Nakata, "PD-L1 overexpression is partially regulated by EGFR/HER2 signaling and associated with poor prognosis in patients with non-small-cell lung cancer," Cancer Immunology, Immunotherapy, vol. 66, no. 7, pp. 865-876, 2017.

[21] D. Kazandjian, D. L. Suzman, G. Blumenthal et al., "FDA approval summary: nivolumab for the treatment of metastatic non-small cell lung cancer with progression on or after platinum-based chemotherapy," The Oncologist, vol. 21, no. 5, pp. 634-642, 2016.

[22] M. Skrzypski and J. Jassem, "Consolidation systemic treatment after radiochemotherapy for unresectable stage III non-small cell lung cancer," Cancer Treatment Reviews, vol. 66, pp. 114-121, 2018.

[23] J. Brahmer, K. L. Reckamp, P. Baas et al., "Nivolumab versus docetaxel in advanced squamous-cell non-small-cell lung cancer," The New England Journal of Medicine, vol. 373, no. 2, pp. 123-135, 2015.

[24] R. S. Herbst, P. Baas, D. W. Kim et al., "Pembrolizumab versus docetaxel for previously treated, PD-L1-positive, advanced non-small-cell lung cancer (KEYNOTE-010): a randomised controlled trial," The Lancet, vol. 387, no. 10027, pp. 1540-1550, 2016.

[25] M. Huang, Y. Lou, J. Pellissier et al., "Cost-effectiveness of pembrolizumab versus docetaxel for the treatment of previously treated PD-L1 positive advanced NSCLC patients in the United States," Journal of Medical Economics, vol. 20, no. 2, pp. 140-150, 2017.

[26] R. Büttner, J. R. Gosney, B. Skov et al., "Programmed deathligand 1 immunohistochemistry testing: a review of analytical assays and clinical implementation in non-small-cell lung cancer," Journal of Clinical Oncology, vol. 35, no. 34, pp. 3867-3876, 2017.

[27] M. Hersom and J. T. Jorgensen, "Companion and complementary diagnostics-focus on PD-L1 expression assays for PD-1/PD-L1 checkpoint inhibitors in non-small cell lung cancer," Therapeutic Drug Monitoring, vol. 40, no. 1, pp. 9-16, 2017.

[28] M. A. J. Iafolla and R. A. Juergens, "Update on programmed death-1 and programmed death-ligand 1 inhibition in the treatment of advanced or metastatic non-small cell lung cancer," Frontiers in Oncology, vol. 7, p. 67, 2017.

[29] J. Cogswell, H. D. Inzunza, Q. Wu et al., "An analytical comparison of Dako 28-8 pharmDx assay and an E1L3N laboratory-developed test in the immunohistochemical detection of programmed death-ligand 1," Molecular Diagnosis \& Therapy, vol. 21, no. 1, pp. 85-93, 2017.

[30] T. Phillips, P. Simmons, H. D. Inzunza et al., "Development of an automated PD-L1 immunohistochemistry (IHC) assay for non-small cell lung cancer," Applied Immunohistochemistry \& Molecular Morphology, vol. 23, no. 8, pp. 541-549, 2015.

[31] A. H. Scheel and S. C. Schäfer, "Current PD-L1 immunohistochemistry for non-small cell lung cancer," Journal of Thoracic Disease, vol. 10, no. 3, pp. 1217-1219, 2018.

[32] D. Fujimoto, Y. Sato, K. Uehara et al., "Predictive performance of four programmed cell death ligand 1 assay systems on nivolumab response in previously treated patients with non-small cell lung cancer," Journal of Thoracic Oncology, vol. 13, no. 3, pp. 377-386, 2018.

[33] S. Hendry, D. J. Byrne, G. M. Wright et al., "Comparison of four PD-L1 immunohistochemical assays in lung cancer," Journal of Thoracic Oncology, vol. 13, no. 3, pp. 367-376, 2018.

[34] D. P. Clark, "Biomarkers for immune checkpoint inhibitors: the importance of tumor topography and the challenges to cytopathology," Cancer Cytopathology, vol. 126, no. 1, pp. 11-19, 2018.

[35] N. A. Rizvi, M. D. Hellmann, A. Snyder et al., "Mutational landscape determines sensitivity to PD-1 blockade in nonsmall cell lung cancer," Science, vol. 348, no. 6230, pp. 124$128,2015$.

[36] D. T. Le, J. N. Durham, K. N. Smith et al., "Mismatch repair deficiency predicts response of solid tumors to PD-1 blockade," Science, vol. 357, no. 6349, pp. 409-413, 2017.

[37] M. D. Hellmann, T. E. Ciuleanu, A. Pluzanski et al., "Nivolumab plus ipilimumab in lung cancer with a high tumor mutational burden," The New England Journal of Medicine, vol. 378, no. 22, pp. 2093-2104, 2018.

[38] M. D. Hellmann, N. A. Rizvi, J. W. Goldman et al., "Nivolumab plus ipilimumab as first-line treatment for advanced non-small-cell lung cancer (CheckMate 012): results of an open-label, phase 1, multicohort study," The Lancet Oncology, vol. 18 , no. 1 , pp. 31-41, 2017.

[39] A. Jeanson and F. Barlesi, "MEDI 4736 (durvalumab) in nonsmall cell lung cancer," Expert Opinion on Biological Therapy, vol. 17, no. 10, pp. 1317-1323, 2017.

[40] S. Peters, S. Gettinger, M. L. Johnson et al., "Phase II trial of atezolizumab as first-line or subsequent therapy for patients with programmed death-ligand 1-selected advanced nonsmall-cell lung cancer (BIRCH)," Journal of Clinical Oncology, vol. 35, no. 24, pp. 2781-2789, 2017.

[41] S. Igawa, Y. Sato, S. Ryuge et al., "Impact of PD-L1 expression in patients with surgically resected non-small-cell lung cancer," Oncology, vol. 92, no. 5, pp. 283-290, 2017.

[42] T. Shukuya and D. P. Carbone, "Predictive markers for the efficacy of anti-PD-1/PD-L1 antibodies in lung cancer," Journal of Thoracic Oncology, vol. 11, no. 7, pp. 976-988, 2016.

[43] V. Anagnostou, K. N. Smith, P. M. Forde et al., "Evolution of neoantigen landscape during immune checkpoint blockade in non-small cell lung cancer," Cancer Discovery, vol. 7, no. 3, pp. 264-276, 2017.

[44] S. J. Antonia, A. Villegas, D. Daniel et al., "Durvalumab after chemoradiotherapy in stage III non-small-cell lung cancer," The New England Journal of Medicine, vol. 377, no. 20, pp. 1919-1929, 2017. 
[45] G. Mazzaschi, D. Madeddu, A. Falco et al., "Low PD-1 expression in cytotoxic $\mathrm{CD}^{+}$tumor-infiltrating lymphocytes confers an immune-privileged tissue microenvironment in NSCLC with a prognostic and predictive value," Clinical Cancer Research, vol. 24, no. 2, pp. 407-419, 2018.

[46] K. R. Voong, J. Feliciano, D. Becker, and B. Levy, "Beyond PD-L1 testing-emerging biomarkers for immunotherapy in non-small cell lung cancer," Annals of Translational Medicine, vol. 5, no. 18, p. 376, 2017.

[47] B. D. Qin, X. D. Jiao, and Y. S. Zang, "Tumor mutation burden to tumor burden ratio and prediction of clinical benefit of anti-PD-1/PD-L1 immunotherapy," Medical Hypotheses, vol. 116, pp. 111-113, 2018.

[48] M. F. Sanmamed, J. L. Perez-Gracia, K. A. Schalper et al., "Changes in serum interleukin-8 (IL-8) levels reflect and predict response to anti-PD-1 treatment in melanoma and non-small-cell lung cancer patients," Annals of Oncology, vol. 28, no. 8, pp. 1988-1995, 2017.

[49] M. D. Hellmann, T. Nathanson, H. Rizvi et al., "Genomic features of response to combination immunotherapy in patients with advanced non-small-cell lung cancer," Cancer Cell, vol. 33, no. 5, pp. 843-852.e4, 2018.

[50] J. R. Brahmer, C. G. Drake, I. Wollner et al., "Phase I study of single-agent anti-programmed death-1 (MDX-1106) in refractory solid tumors: safety, clinical activity, pharmacodynamics, and immunologic correlates," Journal of Clinical Oncology, vol. 28, no. 19, pp. 3167-3175, 2010.

[51] S. L. Topalian, F. S. Hodi, J. R. Brahmer et al., "Safety, activity, and immune correlates of anti-PD-1 antibody in cancer," The New England Journal of Medicine, vol. 366, no. 26, pp. 2443$2454,2012$.

[52] J. R. Brahmer, S. S. Tykodi, L. Q. M. Chow et al., "Safety and activity of anti-PD-L1 antibody in patients with advanced cancer," The New England Journal of Medicine, vol. 366, no. 26, pp. 2455-2465, 2012.

[53] H. Borghaei, L. Paz-Ares, L. Horn et al., "Nivolumab versus docetaxel in advanced nonsquamous non-small-cell lung cancer," The New England Journal of Medicine, vol. 373, no. 17, pp. 1627-1639, 2015.

[54] A. I. Spira, K. Park, J. Mazières et al., "Efficacy, safety and predictive biomarker results from a randomized phase II study comparing MPDL3280A vs docetaxel in 2L/3L NSCLC (POPLAR)," Journal of Clinical Oncology, vol. 33, 2015.

[55] L. Pai-Scherf, G. M. Blumenthal, H. Li et al., "FDA approval summary: pembrolizumab for treatment of metastatic nonsmall cell lung cancer: first-line therapy and beyond," The Oncologist, vol. 22, no. 11, pp. 1392-1399, 2017.

[56] Z. Sheng, X. Zhu, Y. Sun, and Y. Zhang, "The efficacy of antiPD-1/PD-L1 therapy and its comparison with EGFR-TKIs for advanced non-small-cell lung cancer," Oncotarget, vol. 8, no. 34, pp. 57826-57835, 2017.

[57] G. W. Zhou, Y. Xiong, S. Chen, F. Xia, Q. Li, and J. Hu, "Anti-PD-1/PD-L1 antibody therapy for pretreated advanced nonsmall-cell lung cancer: a meta-analysis of randomized clinical trials," Medicine, vol. 95, no. 35, article e4611, 2016.

[58] I. Attili, A. Passaro, A. Pavan, P. F. Conte, F. De Marinis, and L. Bonanno, "Combination immunotherapy strategies in advanced non-small cell lung cancer (NSCLC): does biological rationale meet clinical needs?," Critical Reviews in Oncology/Hematology, vol. 119, pp. 30-39, 2017.
[59] P. S. Chowdhury, K. Chamoto, and T. Honjo, "Combination therapy strategies for improving PD-1 blockade efficacy: a new era in cancer immunotherapy," Journal of Internal Medicine, vol. 283, no. 2, pp. 110-120, 2018.

[60] M. E. Marmarelis and C. Aggarwal, "Combination immunotherapy in non-small cell lung cancer," Current Oncology Reports, vol. 20, no. 7, p. 55, 2018.

[61] I. Moya-Horno, S. Viteri, N. Karachaliou, and R. Rosell, "Combination of immunotherapy with targeted therapies in advanced non-small cell lung cancer (NSCLC)," Therapeutic Advances in Medical Oncology, vol. 10, 2018.

[62] S. Niyongere, A. Saltos, and J. E. Gray, "Immunotherapy combination strategies (non-chemotherapy) in non-small cell lung cancer," Journal of Thoracic Disease, vol. 10, Supplement 3, pp. S433-S450, 2018.

[63] K. Azuma, K. Ota, A. Kawahara et al., "Association of PD-L1 overexpression with activating EGFR mutations in surgically resected nonsmall-cell lung cancer," Annals of Oncology, vol. 25, no. 10, pp. 1935-1940, 2014.

[64] P. M. Ellis, E. T. Vella, and Y. C. Ung, "Immune checkpoint inhibitors for patients with advanced non-small-cell lung cancer: a systematic review," Clinical Lung Cancer, vol. 18, no. 5, pp. 444-459.e1, 2017.

[65] J. H. Kim, H. S. Kim, and B. J. Kim, "Prognostic value of KRAS mutation in advanced non-small-cell lung cancer treated with immune checkpoint inhibitors: a meta-analysis and review," Oncotarget, vol. 8, no. 29, pp. 48248-48252, 2017.

[66] C. Lin, X. Chen, M. Li et al., "Programmed death-ligand 1 expression predicts tyrosine kinase inhibitor response and better prognosis in a cohort of patients with epidermal growth factor receptor mutation-positive lung adenocarcinoma," Clinical Lung Cancer, vol. 16, no. 5, pp. e25-e35, 2015.

[67] Y. Tang, W. Fang, Y. Zhang et al., "The association between PD-L1 and EGFR status and the prognostic value of PD-L1 in advanced non-small cell lung cancer patients treated with EGFR-TKIs," Oncotarget, vol. 6, no. 16, pp. 14209-14219, 2015.

[68] A. D'Incecco, M. Andreozzi, V. Ludovini et al., "PD-1 and PD-L1 expression in molecularly selected non-small-cell lung cancer patients," British Journal of Cancer, vol. 112, no. 1, pp. 95-102, 2015.

[69] E. A. Akbay, S. Koyama, J. Carretero et al., "Activation of the PD-1 pathway contributes to immune escape in EGFR-driven lung tumors," Cancer Discovery, vol. 3, no. 12, pp. 1355-1363, 2013.

[70] S. N. Gettinger, L. Horn, L. Gandhi et al., "Long-term survival, clinical activity, and safety of nivolumab (anti-PD-1; BMS-936558, ONO-4538) in patients (Pts) with advanced non-small cell lung cancer (NSCLC)," International Journal of Radiation Oncology, Biology, Physics, vol. 90, no. 5, pp. S34-S35, 2014.

[71] D. L. Gibbons, L. Q. Chow, D. W. Kim et al., "57O Efficacy, safety and tolerability of MEDI4736 (durvalumab [D]), a human IgG1 anti-programmed cell death-ligand-1 (PD-L1) antibody, combined with gefitinib $(\mathrm{G})$ : a phase I expansion in TKI-naïve patients (pts) with EGFR mutant NSCLC," Journal of Thoracic Oncology, vol. 11, no. 4, article S79, 2016.

[72] M. J. Ahn, J. Yang, H. Yu et al., "136O: Osimertinib combined with durvalumab in EGFR-mutant non-small cell lung 
cancer: results from the TATTON phase Ib trial," Journal of Thoracic Oncology, vol. 11, no. 4, article S115, 2016.

[73] J. J. Han, D. W. Kim, J. Koh et al., "Change in PD-L1 expression after acquiring resistance to gefitinib in EGFR-mutant non-small-cell lung cancer," Clinical Lung Cancer, vol. 17, no. 4, pp. 263-270.e2, 2016.

[74] K. Haratani, H. Hayashi, T. Tanaka et al., "Tumor immune microenvironment and nivolumab efficacy in EGFR mutation-positive non-small-cell lung cancer based on T790M status after disease progression during EGFR-TKI treatment," Annals of Oncology, vol. 28, no. 7, pp. 15321539, 2017.

[75] L. Jiang, X. Su, T. Zhang et al., "PD-L1 expression and its relationship with oncogenic drivers in non-small cell lung cancer (NSCLC)," Oncotarget, vol. 8, no. 16, pp. 26845-26857, 2017.

[76] D. Rangachari, P. A. VanderLaan, M. Shea et al., "Correlation between classic driver oncogene mutations in EGFR, ALK, or ROS1 and 22C3-PD-L1 $\geq 50 \%$ expression in lung adenocarcinoma," Journal of Thoracic Oncology, vol. 12, no. 5, pp. 878-883, 2017.

[77] R. A. Soo, S. M. Lim, N. L. Syn et al., "Immune checkpoint inhibitors in epidermal growth factor receptor mutant nonsmall cell lung cancer: current controversies and future directions," Lung Cancer, vol. 115, pp. 12-20, 2018.

[78] L. Apetoh, S. Ladoire, G. Coukos, and F. Ghiringhelli, "Combining immunotherapy and anticancer agents: the right path to achieve cancer cure?," Annals of Oncology, vol. 26, no. 9, pp. 1813-1823, 2015.

[79] M. Qiao, T. Jiang, S. Ren, and C. Zhou, "Combination strategies on the basis of immune checkpoint inhibitors in nonsmall-cell lung cancer: where do we stand?," Clinical Lung Cancer, vol. 19, no. 1, pp. 1-11, 2018.

[80] C. J. Langer, S. M. Gadgeel, H. Borghaei et al., "Carboplatin and pemetrexed with or without pembrolizumab for advanced, non-squamous non-small-cell lung cancer: a randomised, phase 2 cohort of the open-label KEYNOTE-021 study," The Lancet Oncology, vol. 17, no. 11, pp. 1497-1508, 2016.

[81] N. A. Rizvi, M. D. Hellmann, J. R. Brahmer et al., "Nivolumab in combination with platinum-based doublet chemotherapy for first-line treatment of advanced non-small-cell lung cancer," Journal of Clinical Oncology, vol. 34, no. 25, pp. 2969-2979, 2016.

[82] S. V. Liu, J. D. Powderly, D. R. Camidge et al., "Safety and efficacy of MPDL3280A (anti-PDL1) in combination with platinum-based doublet chemotherapy in patients with advanced non-small cell lung cancer (NSCLC)," Journal of Clinical Oncology, vol. 33, 2015.

[83] L. Gandhi, D. Rodríguez-Abreu, S. Gadgeel et al., "Pembrolizumab plus chemotherapy in metastatic non-small-cell lung cancer," The New England Journal of Medicine, vol. 378, no. 22, pp. 2078-2092, 2018.

[84] M. A. Socinski, R. M. Jotte, F. Cappuzzo et al., "Overall survival (OS) analysis of IMpower150, a randomized $\mathrm{Ph} 3$ study of atezolizumab (atezo) + chemotherapy (chemo) \pm bevacizumab (bev) vs chemo + bev in $1 \mathrm{~L}$ nonsquamous (NSQ) NSCLC," Journal of Clinical Oncology, vol. 36, 2018.

[85] F. G. Herrera, J. Bourhis, and G. Coukos, "Radiotherapy combination opportunities leveraging immunity for the next oncology practice," CA: A Cancer Journal for Clinicians, vol. 67 , no. 1 , pp. $65-85,2017$.
[86] R. A. Chandra, T. J. Wilhite, T. A. Balboni et al., “A systematic evaluation of abscopal responses following radiotherapy in patients with metastatic melanoma treated with ipilimumab," OncoImmunology, vol. 4, no. 11, article e1046028, 2015.

[87] K. R. Voong, J. Naidoo, and D. S. Ettinger, “The next frontier in non-small cell lung cancer: synergizing radiation therapy and immune checkpoint blockade," Clinical Advances in Hematology \& Oncology, vol. 15, no. 8, pp. 615-625, 2017.

[88] K. A. Ahmed, B. C. Creelan, S. Kim, T. J. Dilling, and B. A. Perez, "Safety and tolerability of extracranial radiation therapy and immune checkpoint inhibitors among patients with metastatic non-small cell lung cancer," International Journal of Radiation Oncology, Biology, Physics, vol. 96, no. 2, article S201, 2016.

[89] T. J. Bledsoe, C. E. Rutter, N. H. Lester-Coll, X. Bi, and R. H. Decker, "Radiation to oligoprogessive sites of disease can prolong the duration of response to immune checkpoint inhibitors in patients with metastatic non-small cell lung cancer," International Journal of Radiation Oncology, Biology, Physics, vol. 96, no. 2, article E479, 2016.

[90] R. C. Walshaw, J. Honeychurch, and T. M. Illidge, "Stereotactic ablative radiotherapy and immunotherapy combinations: turning the future into systemic therapy?," The British Journal of Radiology, vol. 89, no. 1066, 2016.

[91] S. Takamori, G. Toyokawa, K. Takada, F. Shoji, T. Okamoto, and Y. Maehara, "Combination therapy of radiotherapy and anti-PD-1/PD-L1 treatment in non-small-cell lung cancer: a mini-review," Clinical Lung Cancer, vol. 19, no. 1, pp. 12-16, 2018.

[92] M. Khunger, P. Jain, S. Rakshit et al., "Safety and efficacy of PD-1/PD-L1 inhibitors in treatment-naive and chemotherapy-refractory patients with non-small-cell lung cancer: a systematic review and meta-analysis," Clinical Lung Cancer, vol. 19, no. 3, pp. e335-e348, 2018.

[93] N. S. McCall, A. P. Dicker, and B. Lu, "Beyond concurrent chemoradiation: the emerging role of $\mathrm{PD}-1 / \mathrm{PD}-\mathrm{L} 1$ inhibitors in stage III lung cancer," Clinical Cancer Research, vol. 24, no. 6, pp. 1271-1276, 2018.

[94] T. Kordbacheh, J. Honeychurch, F. Blackhall, C. Faivre-Finn, and T. Illidge, "Radiotherapy and anti-PD-1/PD-L1 combinations in lung cancer: building better translational research platforms," Annals of Oncology, vol. 29, no. 2, pp. 301-310, 2018.

[95] R. S. Herbst, J. Martin-Liberal, E. Calvo et al., "Interim safety and clinical activity in patients with advanced NSCLC from a multi-cohort phase 1 study of ramucirumab (R) plus pembrolizumab (P)," Annals of Oncology, vol. 27, Supplement 6, 2016.

[96] C. Manegold, A.-M. C. Dingemans, J. E. Gray et al., "The potential of combined immunotherapy and antiangiogenesis for the synergistic treatment of advanced NSCLC," Journal of Thoracic Oncology, vol. 12, no. 2, pp. 194-207, 2017.

[97] E. Vacchelli, F. Aranda, A. Eggermont et al., "Trial watch: IDO inhibitors in cancer therapy," OncoImmunology, vol. 3, no. 10, article e957994, 2014.

[98] T. C. Gangadhar, O. Hamid, D. C. Smith et al., "Epacadostat plus pembrolizumab in patients with advanced melanoma and select solid tumors: updated phase 1 results from ECHO202/KEYNOTE-037," Ann Oncol, vol. 27, Supplement 6, 2016.

[99] AACR, "Epacadostat shows value in two SCCHN trials," Cancer Discovery, vol. 7, no. 9, article OF2, 2017. 
[100] L. Paz-Ares, J. Brahmer, M. D. Hellmann et al., "144TiPCheckMate 227: a randomized, open-label phase 3 trial of nivolumab, nivolumab plus ipilimumab, or nivolumab plus chemotherapy versus chemotherapy in chemotherapy-naïve patients with advanced non-small cell lung cancer (NSCLC)," Annals of Oncology, vol. 28, Supplement 2, 2017.

[101] J. S. Yi, N. Ready, P. Healy et al., "Immune activation in early-stage non-small cell lung cancer patients receiving neoadjuvant chemotherapy plus ipilimumab," Clinical Cancer Research, vol. 23, no. 24, pp. 7474-7482, 2017.

[102] L. J. Rupp, K. Schumann, K. T. Roybal et al., "CRISPR/Cas9mediated PD-1 disruption enhances anti-tumor efficacy of human chimeric antigen receptor T cells," Scientific Reports, vol. 7, no. 1, p. 737, 2017.

[103] S. Antonia, S. B. Goldberg, A. Balmanoukian et al., "Safety and antitumour activity of durvalumab plus tremelimumab in non-small cell lung cancer: a multicentre, phase 1b study," The Lancet Oncology, vol. 17, no. 3, pp. 299-308, 2016.

[104] M. A. Gubens, L. V. Sequist, J. Stevenson et al., "Phase I/II study of pembrolizumab (pembro) plus ipilimumab (ipi) as second-line therapy for NSCLC: KEYNOTE-021 cohorts D and H," Journal of Clinical Oncology, vol. 34, 2016.

[105] H. F. Tsai and P. N. Hsu, "Cancer immunotherapy by targeting immune checkpoints: mechanism of T cell dysfunction in cancer immunity and new therapeutic targets," Journal of Biomedical Science, vol. 24, no. 1, p. 35, 2017.

[106] L. B. John, C. Devaud, C. P. M. Duong et al., “Anti-PD-1 antibody therapy potently enhances the eradication of established tumors by gene-modified T cells," Clinical Cancer Research, vol. 19, no. 20, pp. 5636-5646, 2013.

[107] S. L. Topalian, C. G. Drake, and D. M. Pardoll, "Immune checkpoint blockade: a common denominator approach to cancer therapy," Cancer Cell, vol. 27, no. 4, pp. 450-461, 2015.

[108] S. Koyama, E. A. Akbay, Y. Y. Li et al., "Adaptive resistance to therapeutic PD-1 blockade is associated with upregulation of alternative immune checkpoints," Nature Communications, vol. 7, article 10501, 2016.

[109] K. Suda, L. Rozeboom, C. J. Rivard et al., “Therapy-induced E-cadherin downregulation alters expression of programmed death ligand-1 in lung cancer cells," Lung Cancer, vol. 109, pp. 1-8, 2017.

[110] F. Passiglia, A. Galvano, S. Rizzo et al., "Looking for the best immune-checkpoint inhibitor in pre-treated NSCLC patients: an indirect comparison between nivolumab, pembrolizumab and atezolizumab," International Journal of Cancer, vol. 142, no. 6, pp. 1277-1284, 2018. 


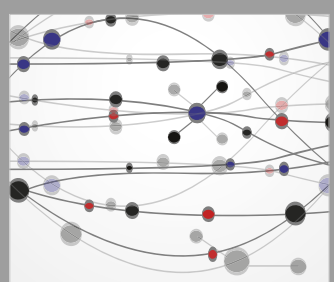

The Scientific World Journal
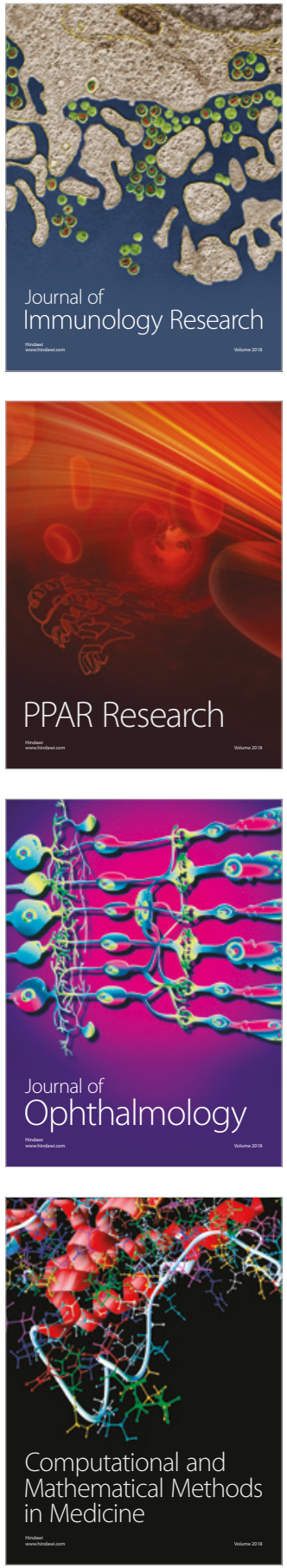

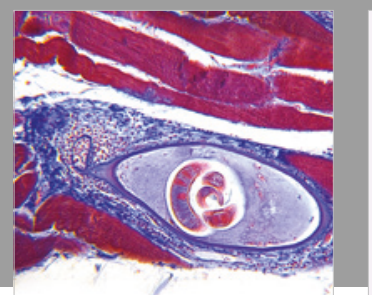

Gastroenterology Research and Practice

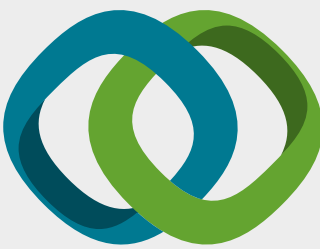

\section{Hindawi}

Submit your manuscripts at

www.hindawi.com
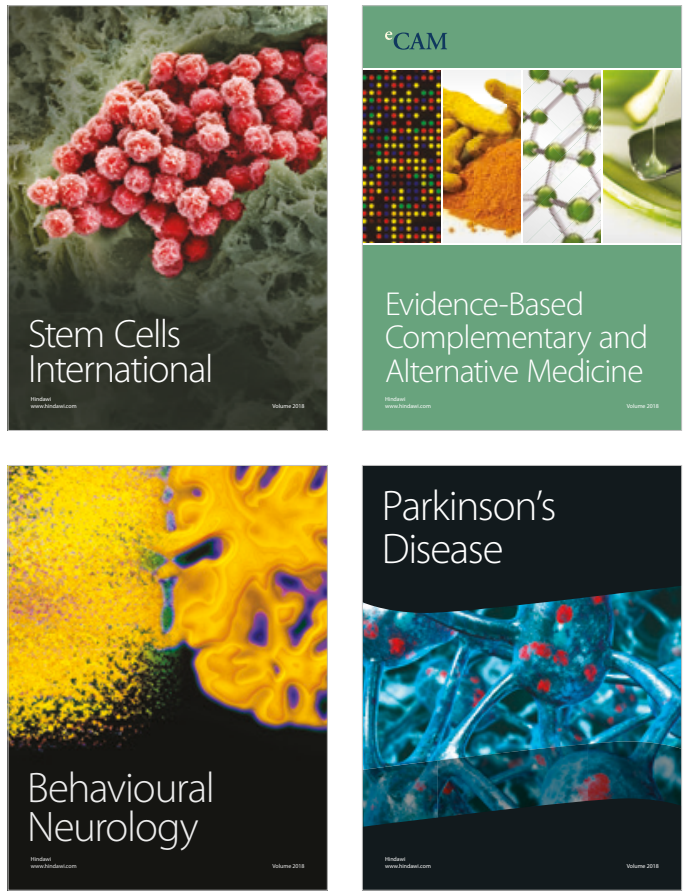

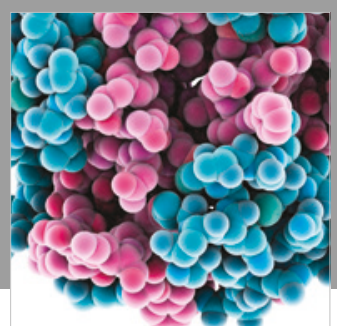

ournal of

Diabetes Research

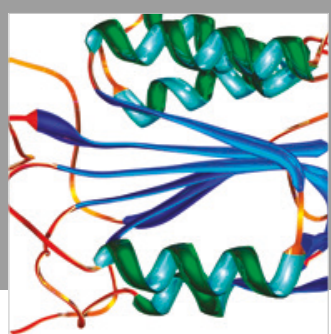

Disease Markers
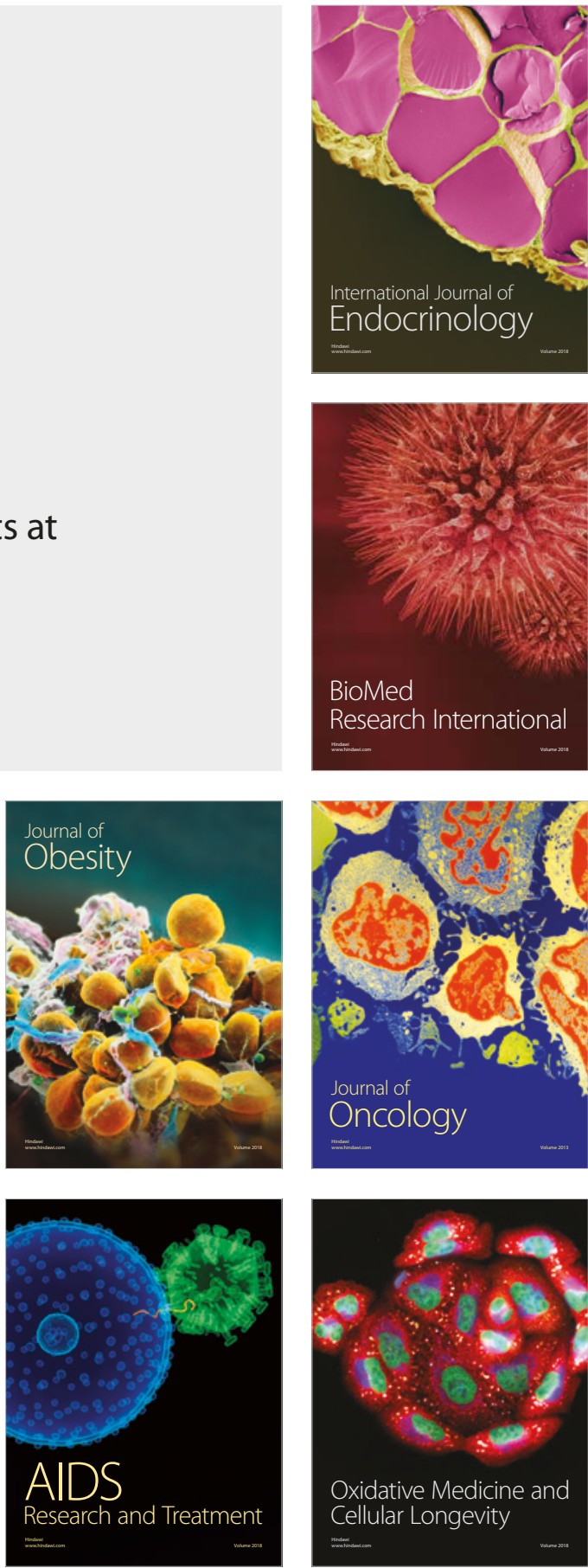